\title{
Intention understanding over T: a neuroimaging study on shared representations and tennis return predictions
}

\author{
Stephanie Cacioppo ${ }^{1,2 *}$, Frederic Fontang ${ }^{3}$, Nisa Patel $^{4}$, Jean Decety ${ }^{5}$, George Monteleone ${ }^{2}$ and \\ John T. Cacioppo ${ }^{1,2,5}$ \\ ${ }^{1}$ Department of Psychiatry and Behavioral Neuroscience, The University of Chicago, Chicago, IL, USA \\ ${ }^{2}$ High-Performance Electrical Neurolmaging Laboratory, Center for Cognitive and Social Neuroscience, The University of Chicago, Chicago, IL, USA \\ 3 Tennis Academy, Pau, France \\ ${ }^{4}$ Department of Graduate Nursing, Western University of Health Sciences, Pomona, CA, USA \\ ${ }^{5}$ Department of Psychology, Brain Imaging Center, The University of Chicago, Chicago, IL, USA
}

Edited by:

Aron K. Barbey, University of Illinois at Urbana-Champaign, USA

\section{Reviewed by:}

Alissa Fourkas, National Institutes of Health, USA

Lucía Amoruso, Laboratory of

Experimental Psychology \&

Neuroscience (LPEN) - INECO

Foundation (FINECO), Argentina

\section{*Correspondence:}

Stephanie Cacioppo,

High-Performance Electrical

Neurolmaging Laboratory, Center

for Cognitive and Social

Neuroscience, The University of

Chicago, 940 East 57 th Street,

Chicago, IL 60637, USA

e-mail:scacioppo@bsd.uchicago.edu
Studying the way athletes predict actions of their peers during fast-ball sports, such as a tennis, has proved to be a valuable tool for increasing our knowledge of intention understanding. The working model in this area is that the anticipatory representations of others' behaviors require internal predictive models of actions formed from pre-established and shared representations between the observer and the actor. This model also predicts that observers would not be able to read accurately the intentions of a competitor if the competitor were to perform the action without prior knowledge of their intention until moments before the action. To test this hypothesis, we recorded brain activity from 25 male tennis players while they performed a novel behavioral tennis intention inference task, which included two conditions: (i) one condition in which they viewed video clips of a tennis athlete who knew in advance where he was about to act/serve (initially intended serves) and (ii) one condition in which they viewed video clips of that same athlete when he did not know where he was to act/serve until the target was specified after he had tossed the ball into the air to complete his serve (non-initially intended serves). Our results demonstrated that (i) tennis expertise is related to the accuracy in predicting where another server intends to serve when that server knows where he intends to serve before (but not after) he tosses the ball in the air; and (ii) accurate predictions are characterized by the recruitment of both cortical areas within the human mirror neuron system (that is known to be involved in higher-order (top-down) processes of embodied cognition and shared representation) and subcortical areas within brain regions involved in procedural memory (caudate nucleus). Interestingly, inaccurate predictions instead recruit areas known to be involved in low-level (bottom-up) computational processes associated with the sense of agency and self-other distinction.

Keywords: embodied cognition, biosocial interaction, dyads, intention understanding, shared representation, fMRI, social neuroscience, mirror neuron system

\section{INTRODUCTION}

"If there is something you don't want to be on a tennis court, it is predictable,"

John McEnroe, ESPN, U.S Open, 8-30-13.

The way in which athletes read and anticipate the actions of their opponent during fast-ball sports, such as a tennis, is a challenging and complex process that is a remarkable feat in itself. A tennis player's ability to predict an opponent's intentions quickly and accurately is particularly important during the return of serves, where the time required to plan and initiate a response typically exceeds the flight time for the ball (Glencross and Cibich, 1977; Farrow and Abernethy, 2003; Williams et al., 2004; Wright and Jackson, 2007). Given the high speed a ball can reach nowadays (e.g., above $130 \mathrm{mph}$ ), the receiver must make a decision regarding the direction of his/her opponent's serve intentions (e.g., to serve to the center of the tennis court or $\mathrm{T}$, the middle of the service box, or the wide side of the service box) based, at least in part, on information identified prior to the server striking the ball (Wright and Jackson, 2007).

Several studies have investigated the mechanisms underlying predictive motor skills in such time-constrained situations (Williams et al., 2004). For instance, it has been shown that expert tennis players, when compared to their less skilled counterparts, are: (i) better at detecting advance (i.e., early or pre-event) information from an opponent's postural orientation (Williams et al., 2002); (ii) have more efficient visual search behaviors (Goulet et al., 1988, 1992; Singer et al., 1996; Helsen and Starkes, 1999; Overney et al., 2008); (iii) pay more attention to motion information (Williams et al., 2002); and (iv) possess greater 
knowledge/expertise of situational probabilities (Williams et al., 2004). Although the past decade has been characterized by a growing body of research dedicated to better understand the factors playing a role in anticipation and predictive skills in fast-ball sports, very few studies have examined their underlying neural mechanisms (e.g., Wright and Jackson, 2007). Among these studies, Wright and Jackson (2007) used temporal occlusion to study the neural bases of action prediction in fast-ball sports. Relative to a passive condition, action prediction recruited notably a frontoparietal network (Wright and Jackson, 2007), which is known to involve the putative human mirror neuron system (hMNS; Grafton et al., 1996; Rizzolatti and Sinigaglia, 2008; Rizzolatti and Fogassi, 2014). They extended this result by demonstrating that experts, compared to novices, tend to show stronger brain activation within the hMNS for early-occluded than for late-occluded time sequences of a tennis shot (Wright and Jackson, 2007).

Theories of simulation and embodied cognition provide a neural basis for such early predictive ability in experts by specifying the involvement (and re-activation) of the inferior fronto-parietal network (possibly including the hMNS), which is known to be activated by one's own motor performance as well as perspective taking, sensorimotor integration, and procedural memory (Rizzolatti et al., 1996; Blakemore and Decety, 2001; Ruby and Decety, 2001; Ruby et al., 2002; Buccino et al., 2004; Rizzolatti and Sinigaglia, 2007, 2008; Grafton, 2009; Grafton et al., 2009; Ortigue et al., 2009a,b, 2010a; Juan et al., 2013; Tomeo et al., 2013; Rizzolatti and Fogassi, 2014). Such research has highlighted two overlapping neural networks, known as the action observation network (AON) and the social network $(\mathrm{SN})$, that are differentially involved in the process of understanding intentions and actions (Grafton, 2009; Ortigue et al., 2009a,b, 2010a; Juan et al., 2013). The AON, which contains a subset of regions within the hMNS including the posterior superior temporal sulcus (STS), the inferior parietal lobule, and the inferior frontal gyrus, has been linked to perception of actions and understanding intentions utilizing embodied cognition (Grafton et al., 1996; Desmurget and Grafton, 2000; Rizzolatti and Craighero, 2004; Rizzolatti and Sinigaglia, 2007, 2008; Desmurget et al., 2009). On the other hand, the SN includes the medial prefrontal cortex, STS, precuneus, insula, and amygdala, and has been linked to perceiving biological motion and theory of mind attribution (Frith and Frith, 1999; Allison et al., 2000; Wheatley et al., 2007; Decety and Cacioppo, 2012). Theories of embodied cognition and simulation suggest that the emulation of these two brain networks contributes to the capacity to read and predict the intentions of others.

Although embodied cognition is not a prerequisite to act or to understand others' actions, simulation theories suggest that the more these observed actions are congruent with integrated templates of past self-related motor experiences, the easier it is to read these observed actions and intentions as the actor and the observer share a mental map of the action (Niedenthal et al., 2005; Niedenthal, 2007). In line with this, the model of shared representation suggests that sport mates or close partners develop a "transactive" mental representation of their self while acinga mental representation that calls for cognitive interdependence and includes a structure of stored information across the two individuals (Wegner et al., 1985; Ortigue et al., 2010b). Cognitive interdependence in dyads relates to the concept of inclusion of the other in the self-mental representation-a concept that is closely tied to self-expansion mechanisms, and embodied cognition. Although we are all interdependent to some degree, the model of shared representation highlights the extent to which partners may implicitly read and influence each other's perceptions of their actions, emotions, and intentions. Cognitive interdependence can provide a processing advantage during anticipatory representations of others' behaviors, such that sport mates (e.g., competitors) are more efficient and more frequently successful in forming shared mental representations based on internal predictive models of actions (Ruscher et al., 2003).

In the context of sport athletes, intention understanding among peers is based, in part, upon a shared mental representation of actions, with sport mates being able to better anticipate one another's actions due to greater experience observing each other's actions in different situations or due to shared experience in a specific sport (Wegner et al., 1985; Hommel et al., 2001; Ruscher et al., 2003; Agnew and Etcheverry, 2006). As an illustrative case in point, previous studies have demonstrated that experts in a sport (e.g., basketball, dance, or soccer) are better and/or faster (than novices) at understanding intentions of an opponent or a teammate just by watching their body movements (Jeannerod, 2001; Wolpert et al., 2003; Calvo-Merino et al., 2005; Cross et al., 2006, 2009; Aglioti et al., 2008; Abreu et al., 2012; Tomeo et al., 2013). Moreover, evidence suggests that intention perception may be facilitated not only by congruence between observed and past actions (Niedenthal et al., 2005; Niedenthal, 2007; Aglioti et al., 2008; Ortigue et al., 2010a,b; Tomeo et al., 2013), but also by the emotional bond between actor and perceiver, with a stronger bond associated with better (Cutting and Kozlowski, 1977) or faster intention understanding (Ortigue et al., 2010b). This facilitation effect putatively occurs through a direct, fast and automatic visuo-motor matching process between what the experts see and what they have executed (over and over) in the past.

Reciprocally, this model of shared representation predicts that this facilitation effect fades away when an actor plans their actions with an unusual mental representation of their intentions (as it can be the case in fool actions). In a recent behavioral study, Tomeo and colleagues tested this notion by manipulating the congruence between a soccer kicker's bodily movements and the subsequent ball trajectory and investigated the prediction performance from 16 kickers, 16 goalkeepers, and 16 novices (Experiment 1; Tomeo et al., 2013). Their results showed that kickers were more often fooled than goalkeepers and novices during incongruent actions, although both types of experts (kickers and goalkeepers) outperformed novices (Tomeo et al., 2013). This study reinforced the model of shared representation by demonstrating that: (i) shared expertise plays a crucial role in intention anticipation (which has also been demonstrated previously by a large body of work), and that (ii) previously shared mental representation among peers (kickers-to-kickers) may hinder intention anticipation when one of them performs deceptive actions. However, because an actor always knows in advance what 
their intentions are (whether they are deceptive intentions or not) and so may display visual cues that are shared with their observers, one may argue that this study does not fully test the model of shared intentions.

To address this argument, we designed a novel behavioral intention inference task (IIT), which included two conditions: (i) one condition showing video clips of an unfamiliar right-handed tennis athlete (hereafter the server) who knew in advance where he was about to act/serve (initially intended action, IIS condition; see Supplementary Movie 1 for a sample) and (ii) another condition showing video clips of that same athlete when he did not know in advance where he was about to act/serve (non-initially intended serve, NIIS condition; see Supplementary Movie 2 for a sample). In this NIIS condition, an experimenter (NP) told the server where to serve (either to the center of the tennis court or to the wide side of the service box) after he had tossed the ball into the air to serve. Although the voice of the experimenter, NP, was recorded in each video for quality and accuracy control purposes, participants watched silent video clips. In all video clips the server bounced the ball twice, tossed the ball, and finally stroke it to perform a series of serves.

We recorded brain activity from 25 male tennis experts (hereafter, the observers) while they were performing this tennis IIT ( $t$ IIT; Figure 1) and were asked to predict the ultimate direction (either to the $\mathrm{T}$ or to the wide side of their service box; see Figure 2 for an illustration of these two serve directions). As in previous studies (Williams et al., 2004), serves that landed in the middle of the service box were not considered, because of the potential difficulty to classify objectively an observer's response to such serves. No feedback was provided to the participants during the $t$ IIT. The participants were not aware that the server was performing this series of serves under the above two different conditions (initially intended serves and non-initially intended serve). Because, the ability to evaluate the body movements of another

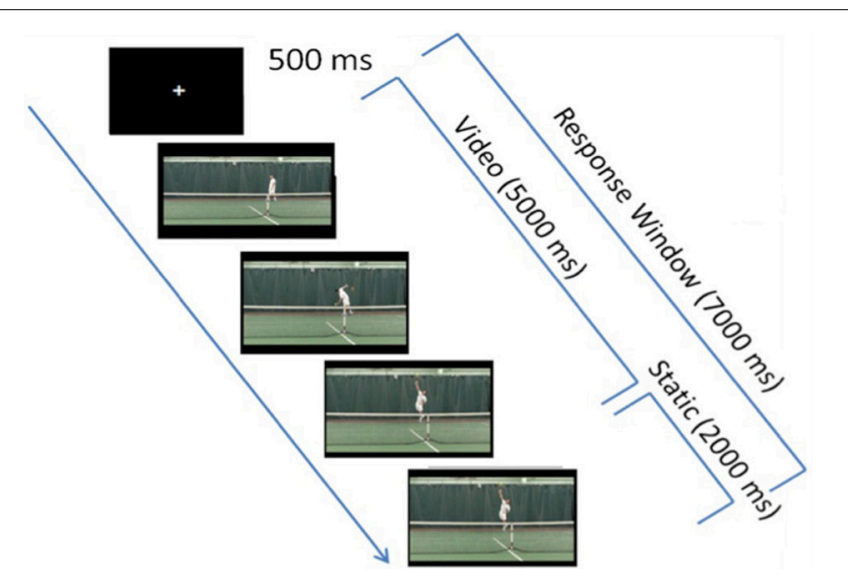

FIGURE 1 | Experimental paradigm. Each trial first consisted of a 500 ms-fixation cross, followed by a 5-s video clip, which froze on the last frame of the video clip (when the player's racquet struck the ball) for an additional $2 \mathrm{~s}$ in order to provide participants with more response time, if needed. In each video clip, the tennis player was shown bouncing the ball twice, then winding up and striking the ball. player and predict their intentions is facilitated by coupling previous experiences (both the sensorimotor cues that diagnose serve location and the sensorimotor cues that do not) with any sensory input (e.g., visual cues) that might cue them as to the intended action (Kording and Wolpert, 2004; Kilner and Frith, 2007; Kilner et al., 2007), we assumed that, in the absence of reliable sensory information due, for instance, to the high speed of a tennis serve, expert tennis players would rely more on their previous bodily visuo-motor experiences to determine the intended location of the serve than visual information (Kording and Wolpert, 2004). It also follows that when an opponent completes most of the action (e.g., a tennis serve) without any clear intention in mind, expert tennis players should be near or at chance level when trying to predict the direction of that action.

Interestingly, in the case of a tennis match, the same action (e.g., a tennis serve) may reflect different intentions (e.g., to serve to the $\mathrm{T}$ or the wide side of the service box). Given the importance of not being predictable on the tennis court, expert tennis players are taught to perform the same perceptible actions regardless of their service intentions. Therefore, expert tennis players not only have to rely on their past experiences of serving in tennis to predict the intended location of their opponent's serve but they must eschew any masking behavioral cues that would hide the intention of their opponent. The study of expert tennis players while they try to predict accurately the ultimate intentions (direction of a serve) of another player, thus, constitutes a unique and ecologically valid opportunity to better understand the mechanisms underlying the assumed visuo-motor matching in embodied cognition.

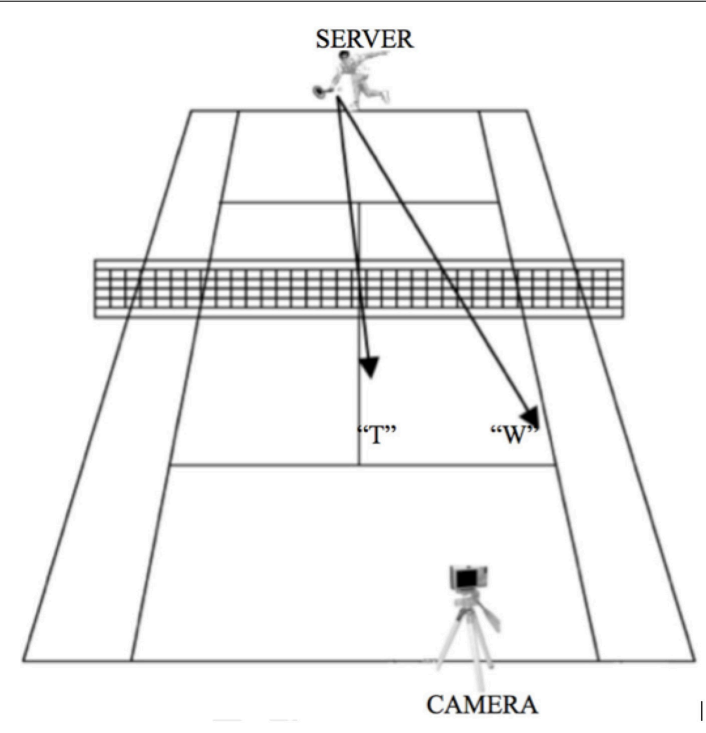

FIGURE 2 | Experimental layout during creation of the stimuli. The camera was located on a tripod on the baseline next to the service box of the court diagonal from where the tennis player stood. The tennis player could serve either to the center of the opposite side of the tennis court $\left(" T^{\prime \prime}\right)$ or to the wide side of the service box ("W"). This setting allowed the participants to have a first person view while watching the video clips, as if they were in a match situation standing on the tennis court ready to receive the serve. 


\section{MATERIALS AND METHODS PARTICIPANTS}

All 25 fMRI participants were right-handed (Edinburgh Handedness Inventory, Oldfield, 1971), and had normal or corrected to-normal visual acuity with no history of psychiatric or neurological disorder (as ascertained by a brief anamnesis and self-report questionnaires, Davis, 1980; Zigmond and Snaith, 1983; Spielberger, 1987; Russell, 1996; Bellini et al., 2002; Lamm et al., 2007). Mean age of participants was 26.2 years $(S D=8.95)$. Because the goal of this study was to test the theory of shared representation among experts, all participants were expert tennis players (see questionnaire section below for further details).

\section{PROCEDURE}

Prior to participation, volunteers provided written informed consent that had been approved by the Ethical Committee of the University of Chicago, Illinois. The study took place over a single visit. Upon arriving at the University of Chicago Brain Imaging Center, participants completed a series of standard screening forms. Then, they completed a series of tennis-related questionnaires, and a brief practice of the tennis intention inference task (practice $t$ IIT) outside the scanner, and an experimental tennis intention inference task (experimental $t \mathrm{IIT}$ ) while they were in the 3T Philips fMRI scanner (see below for further details).

\section{QUESTIONNAIRES}

Participants' tennis expertise was ascertained by their United States Tennis Association (USTA) playing level, which was, on average, $4.24(S D=0.93)$ out of a 7-point scale. A USTA level of 4 denotes a player who has a dependable forehand and backhand, a variety of good shots (such as serves and lobs), and good team work in doubles. To further understand the participants' tennis profile, a series of exploratory questions about their tennis habits/behaviors were also asked to the participants. These additional questions investigated: (a) the number of hours our participants played tennis per week; (b) the number of hours they watched tennis on TV per week; (c) the age when the participants first begun playing tennis; and (d) the number of hours they played tennis on video games per month.

\section{PRACTICE TASK TENNIS INTENTION INFERENCE TASK (PRACTICE TENNIS IIT)}

In the practice $t \mathrm{IIT}$, participants received detailed instructions regarding the task, and viewed one sample video clip for which they were asked to determine the intended location (either to the center of the tennis court i.e., to the $\langle\mathrm{T} \gg$, or to the wide side of the service box i.e., to the $\langle\mathrm{W} \gg$ ) of the serve depicted in that video clip. This example was meant to allow participants to understand fully the instruction they would then perform in the experimental tennis IIT $(t$ IIT).

\section{EXPERIMENTAL TENNIS INTENTION INFERENCE TASK (EXPERIMENTAL $\boldsymbol{t}$ IIT)}

As in the practice $t \mathrm{IIT}$, in the experimental $t$ IIT participants were instructed to indicate as rapidly and as accurately as possible where the tennis server intended to serve (either to the "T" or to the "W" side of the service box).
Each trial began with a $500 \mathrm{~ms}$-fixation cross that was followed immediately by a $5 \mathrm{~s}$-target video clip (Figure 1 ). Because previous research has shown that expert athletes respond to visual cues that occur well before a shot is struck (Ward et al., 2002), all video clips in the $t$ IIT (including video clip used in the practice tIIT) ceased as the expert tennis player's racquet contacted the ball, and remained frozen on this image for a maximum of $2 \mathrm{~s}$ i.e., until the end of the duration of the response window, which started when the video started. As during the brief practice and as in previous IIT studies (Ortigue et al., 2009a,b, 2010a,b), participants were allowed to respond at any point during the video clip presentation, as well as during the $2 \mathrm{~s}$ immediately following the completion of the video clip for a total response window of $7 \mathrm{~s}$ (Figure 1).

\section{STIMULI}

Stimuli consisted of eight video clips [2 types of serves (IIS and NIIS) $\times 2$ starting positions (left and right) $\times 2$ ball landing sides (to the center "T" and to wide side "W" of the service box)] of an unfamiliar right-handed male expert tennis player (from Syracuse University, Upstate New York) performing a tennis serve (one per video). The video clips showed the tennis player on two different starting positions [standing either on the right side from the participant's perspective of the tennis court (half of the video clips), or on the left side of the tennis court] in order to control for any participants' lateralized attentional bias during the experimental $t$ IIT.

Across serve conditions, the server was able to perform the same movements repeatedly, independently of the ultimate outcome of his serves (either to the center or the wide side of the service box) because of his high tennis level (USTA level: 7, which denotes a world class player). He was also able to bounce the ball, toss it and serve in the same way, using his regular action with a relatively consistent velocity, independently of the intentionality manipulation (see Supplementary Movies 1, 2 for examples).

\section{APPARATUS}

Videos of the server were taken with a digital Sony Cybershot camera. The camera was located on a tripod on the baseline next to the service box of the court diagonal from where the tennis player stood (Figure 2). This setting allowed the participants to have a first person view while watching the video clips, as if they were in a match situation standing on the tennis court ready to receive the serve. All video clips were presented using E-Prime 2.0 (Psychology Software Tools Inc., Pittsburgh, USA). During the scanning session, participants viewed the stimuli on a back projection screen mounted on the head coil of the MRI scanner.

\section{VALIDATION OF THE STIMULI AND VIABILITY OF THE PARADIGM}

In order to test the viability of our novel paradigm and test the similarity of the server's movements across serve conditions, we performed three different steps. First, we performed a quantitative analysis of all the tennis video clips using Dartfish i.e., a performance video analysis software. Extensive research on anticipatory skills in sport, in which the visual information available to understand a tennis serve is cut off at some specific time frames (temporal occlusion) during the serve, indicates that a 
key event for tennis is the ball/racket contact, with the movement of the arm and the racket prior that key event being the source of critical cues for racket sports (Tenenbaum et al., 2000; Shim et al., 2006; Abernethy and Zawi, 2007; Jackson and Mogan, 2007; Wright and Jackson, 2007; Williams et al., 2009 for review). Therefore, we analyzed the average speed of the server's movement up until he hit the ball. Results revealed that this average speed did not significantly vary between the IIS and NIIS conditions $\left[M_{I I S}=1.94 \mathrm{~m} / 0.2 \mathrm{~s}, S D=0.13 ; M_{N I I S}=\right.$ $2.03 \mathrm{~m} / 0.2 \mathrm{~s}, S D=0.04 ; t_{(6)}=-1.44 ; p=.20$; two-tailed]. This quantitative analysis revealed that the stimuli from both conditions were visually comparable in terms of speed of the server's movements.

Second, we performed a visual qualitative analysis of the video clips by asking three persons (SC, JTC, BM) who are knowledgeable (although non expert) in tennis to view all the video clips, one by one, and tried to determine whether any obvious visual differences appeared between the two serve conditions i.e., IIS and NIIS. Although these three persons were aware of the two different experimental conditions, none of them was able to identify any visual differences between video clips. This result was reinforced with the behavioral performance from 29 other individuals [ 18 men, 11 women; mean age of $31.55(S D=10.32)$ ] we recruited on Amazon Mechanical Turk (MTurk). To ensure the respondents were real participants, rather than a computerized script, a compliance check question was included that instructed respondents to answer "left" to validate that they were reading the survey prior to responding. Although 50 individuals were initially involved, $42 \%$ of the respondents failed to answer this compliance check correctly, so their data were not included in the analysis. Thus, the final sample was composed of 29 individuals.

All 29 participants were non-expert tennis players (as ascertained by their self-report USTA tennis levels: $M=2.73, S D=$ 1.29) and were not aware of the two serve conditions. Each participant viewed all video clips in one of two specific randomized orders. Results revealed no significant gender difference [for men: $M_{I I S}=51.4 \%, S D=18.1 ; M_{N I I S}=52.8 \%, S D=28.3$; for women: $M_{I I S}=56.8 \%, S D=22.6 ; M_{\text {NIIS serves }}=52.3 \%, S D=$ $28.4 ; t_{(27)}=-0.367$, n.s., $\left.r=-0.073\right]$, and no significant effect of the serve conditions on accuracy $\left[t_{(28)}=0.138, n . s\right.$., $r=$ 0.018], with participants being at chance level for both types of serves $\left(M_{I I S}=53.5 \%, S D=19.7 ; M_{N I I S}=52.6 \%, S D=27.8\right)$. No reaction times were recorded in the MTURK study.

Then, to make sure that this novel task was suitable for tennis experts, we asked a pro-tennis player (FF), who is also an active pro-tennis coach on the ATP tour, to watch the video clips and perform a qualitative analysis. Although he was not aware of the two conditions, he was able to detect nuances at the level of the hips of the server that differed between the two conditions. Interestingly, he was not able to name or identify the two conditions after identifying two different types of stimuli. All he could report was that some tennis serves (the IIS, according to SC's observation of FF's performance) were easier to anticipate than others in the set of video clips. This procedure suggests that a pro-tennis player could not report the specific content (IIS and NIIS) of the video clips, and that we, thus, could use these video clips in our study with tennis experts.

\section{EXPERIMENTAL PARADIGM}

The present $t$ IIT included 5 blocks. Each block was composed of 8 trials. In order to increase the number of stimulus presentations, the participants were asked to perform the experimental $t$ IIT twice: once in the hypothetical context of a friendly match with a tennis practice partner; and once in the hypothetical context of a competitive match with that same tennis practice partner. This procedure led to a total of 10 blocks. Block order was selected at random by E-Prime 2.0 (Psychology Software Tools Inc., Pittsburgh, USA), with the condition that both match types must be presented exactly five times. Accordingly the order of presentation of the 10 blocks differed across participants. All the eight videos were presented once in each of the 10 blocks. The presentation of each video was completely randomized within each of block. In sum, a total 80 trials were presented to each participant.

\section{DEPENDENT MEASURES}

Response accuracy (in percent, \%), reaction times from the onset of the video (in milliseconds, ms), and brain activity were recorded while participants made a decision as to ultimate direction of the serves. In addition, to account for a potential intention advantage, we calculated a conventional accuracy index score (Marshall et al., 1975) for percentage correct responses between Initially Intended Serve (IIS) with Non-Initially Intended Serve (NIIS) condition as follows: (IIS - NIIS)/(IIS + NIIS). Thus, positive values indicate an IIS advantage and negative values a NIIS advantage. Finally, to eliminate any potential response bias in our accuracy measure, we also calculated a $d^{\prime}$ accuracy index (Dprime.AccuracyIndex), using Marshall et al.'s accuracy Index formula as follows: (IIS - NIIS)/(IIS + NIIS). In this $d^{\prime}$ accuracy index, we converted percent correct scores to $d^{\prime}$ for IIS and NIIS items using the formula $\mathrm{z}$ (Hit) $-\mathrm{z}$ (False Alarm), which gave us Dprime.IIS and Dprime.NIIS. Converted Dprime values.

\section{BEHAVIORAL STATISTICAL ANALYSES}

In line with our hypotheses, we collapsed across match hypothetical contexts (friendly or competitive), starting position (left or right) and ball landing sides ("T" or "W"), yielding a repeated-measures design with serve type (IIS vs. NIIS) as a within-subjects factor. Mean reaction times and percentage were calculated for each subject and condition. Outliers were removed by eliminating responses greater than 3.5 standard deviations from the grand mean. Using this cutoff resulted in the removal of $4.5 \%$ of all trials (across participants). Repeated measures ANOVAs were utilized to analyze potential differences in reaction times and accuracy between serve types in the $t$ IIT. Additionally, correlational analyses were performed to examine the relationship between self-reported data about tennis (USTA level, hours playing tennis per week, hours watching tennis on TV per week, and age first learned tennis) and our behavioral dependent measures (See Table 1 for accuracy and reaction times; and see Table S1 for accuracy index and $d^{\prime}$ accuracy index). 
Table 1 | Pearson correlations ( $d f=23$ ) between various tennis-related self-reported measures and behavioral measures.

\begin{tabular}{lrrrrrr}
\hline & $\mathbf{R T}_{\text {all }}$ & $\mathbf{R T}_{\text {IIS }}$ & $\mathbf{R T}_{\text {NIIS }}$ & $\mathbf{A C C}_{\text {all }}$ & $\mathbf{A C C}_{\boldsymbol{I I S}}$ & $\mathbf{A C C}_{\text {NIIS }}$ \\
\hline $\begin{array}{l}\text { USTA level } \\
\text { Hours playing }\end{array}$ & -0.24 & 0.30 & 0.22 & -0.09 & 0.12 & -0.23 \\
$\begin{array}{l}\text { tennis/week } \\
\text { Hours }\end{array}$ & 0.17 & 0.19 & 0.15 & 0.17 & -0.05 & 0.05 \\
$\begin{array}{l}\text { watching } \\
\text { tennis/week }\end{array}$ & & & & & & \\
$\begin{array}{l}\text { Age first } \\
\text { learned tennis }\end{array}$ & -0.03 & -0.06 & -0.004 & -0.31 & -0.26 & -0.23 \\
\end{tabular}

${ }^{*}$ Denotes $p<0.05$.

\section{NEUROIMAGING RECORDINGS AND PROCESSING Magnetic resonance imaging recordings}

Imaging was performed on a 3-T Philips Achieva Quasar Dual 16 Ch scanner with quadrature head coil used for spin excitation and signal reception. High-resolution volumetric T1-weighted spoiled gradient-recalled (SPGR) images were obtained for each participant in one hundred sixty-one $1.0-\mathrm{mm}$ sagittal slices with $8^{\circ} \mathrm{flip}$ angle and $24 \mathrm{~cm}$ field of view (FOV) for use as anatomical images. Functional images using a block design and were acquired using a echo-planar acquisition with Z-Shimming with $32 \times 4$-mm coronal slices with an inter-slice gap of $0.5 \mathrm{~mm}$ spanning the whole brain $\left(T R=2.5 \mathrm{~s}, T E=30 \mathrm{~ms}\right.$, flip angle $=80^{\circ}, \mathrm{FOV}=22 \mathrm{~cm}$, $64 \times 64$ matrix size, fat suppressed).

\section{Functional image processing and analyses}

Image pre-processing and analyses were performed using Analysis of Functional NeuroImages software (AFNI, Medical College of Wisconsin). For each participant, motion detection and correction were undertaken using a six-parameter, rigid-body transformation. Functional images were co-registered and spatially smoothed using a 5-mm full width at half maximum Gaussian filter. Individual-subject analyses were conducted using the general linear model to generate estimates of blood oxygenation leveldependent (BOLD) signal on a voxelwise basis (Ward, 2002). Stimulus timing vectors for each of the experimental conditions were modeled for 3 TRs after each stimulus onset, and each vector was convolved with a gamma-variate waveform using the AFNI program Waver. The resulting model was fit voxelwise to preprocessed time-series data with a linear least-squares model using the AFNI program 3dDeconvolve, generating a map consisting of beta coefficients (fit values) at each voxel for each modeled condition-intended serve/non intended serve; - as well as a baseline coefficient. Two GLMs were assessed for each subjects: one modeling only correctly identified T/W trials, and one modeling only incorrectly identified T/W trials. Output from the deconvolution analysis for each subject was scaled voxelwise to percent signal change from baseline, and each subject's data were spatially transformed to the MNI Colin27 Atlas (1998) stereotaxic coordinate space and interpolated to $3 \mathrm{~mm}^{3}$ isometric voxels for group analysis (Holmes et al., 1998). Voxelwise fMRI analyses were performed at the group level, the results of which were corrected for multiple comparisons by using a Monte Carlo simulation to determine minimum cluster sizes corresponding to an alpha value of 0.05 for voxelwise thresholds of $p<.01$ and $p<.025$ (Nichols, 2012). Groupwise analyses were carried out for correct-item and incorrect-item models. A voxelwise [IIS/NIIS] ANOVA was performed, as well as whole-brain voxelwise Pearson correlation to identify regions in which differential BOLD activity in response to the stimulus effect (i.e., IIS-NIIS) was associated with behavioral responses related to tennis i.e., USTA level, hours playing tennis per week, hours watching tennis on TV per week, and age first learned tennis. In addition, we used the reaction times and intention accuracy index scores (Tables 4, 5) in correlational analyses with the BOLD signal contrasting IIS and NIIS (Voxelwise correlation of BOLD [IIS - NIIS] for correct trials $\times$ Dprime.AccuracyIndex, cluster were also calculate with a threshold at voxelwise $p<.01$ corrected to alpha $<0.05$. See Table S2).

\section{RESULTS}

\section{QUESTIONNAIRES}

On average, participants reported: (a) having a USTA level of 4.24 $(S D=0.93)$ out of a 7-point scale; (b) playing tennis $3.56(S D=$ 3.98) hours per week, (c) watching tennis on TV $1.92(S D=4.31)$ hours per week, and (d) having begun playing tennis at the age of $9.36(S D=5.26)$. Most of the participants also reported not playing tennis on video games (three of the participants reported playing video-tennis game less than 2 hours per month).

\section{Behavioral tIIT results}

As predicted, the behavioral results showed that the observers were better at predicting initially intended serves, IIS (64.16\% correct, $S D=0.099)$ than non-initially intended serves, NIIS $\left[52.40 \%\right.$ correct, $S D=0.099 ; F_{(1,24)}=25.387 ; p<.001 ; d=$ $1.18]$. When percent correct values were converted to $d^{\prime}, \mathrm{Z}(\mathrm{H})-$ $\mathrm{Z}(\mathrm{FA})$, results showed a similar significant effect of IIS/NIIS on accuracy $\left[t_{(24)}=4.736, p=8.126 \mathrm{e}-05 ; 95 \%\right.$ CI $(0.34 ; 0.87)$; mean of $d^{\prime}$ differences $\left.=0.61\right]$. Note that this behavioral result is also statistically significant on the same order of magnitude $(p<.0001)$, when using percent correct in lieu of $d^{\prime}$. No differences in reaction times were observed between the two serve conditions for correct responses $\left[M_{I I S}=5609.64 \mathrm{~ms}, S D=\right.$ $387.58 ; M_{\text {NIIS }}=5637.26 \mathrm{~ms}, S D=425.12 ; F_{(1,24)}=2.62 ; p=$ $.12 ; d=-0.07)$. A post-hoc paired $t$-test conducted to see if reaction times were different between IIS and NIIS for incorrect responses revealed no differences in reaction times between the two serve conditions $\left[M_{I I S}\right.$. incorrect $=5622.75 \mathrm{~ms}, S D=418.99$; $M_{\text {NIIS. } \text { incorrect }}=5636.25 \mathrm{~ms}, S D=438.77 ; t_{(24)}=-0.61 ; p=$ $.55 ; d=-0.031]$. To check whether the participants were able to identify the different conditions during the task, an experimenter $(\mathrm{AB})$ performed a debriefing with each participant after the experimental $t$ IIT. None of the participants reported being aware of the two serve conditions.

\section{Behavioral correlational analyses}

Significant positive correlations were observed between the number of hours the participants reported playing tennis per week and three behavioral measures: (i) the overall accuracy; (ii) the accuracy for IIS; and the $d^{\prime}$ accuracy index. Together these results 
suggest that the more participants reported playing tennis per week, the better they were at predicting accurately the tennis serve of the model, especially when this latter knew in advance where he intended to serve. No other significant correlations were found between the different tennis-related questionnaires and the accuracy or reaction times (See Table 1 and Table S1 for details).

\section{NEUROIMAGING RESULTS IIS-NIIS contrast}

In line with our behavioral results, our neuroimaging results showed regional changes in hemodynamic activity for correct behavioral predictions of initially intended (IIS) serves (compared to non-initially intended, NIIS, serves) in four main cortical areas: right occipital cortex, right superior parietal lobule
(SPL), left extrastriate body area (EBA), and left inferior parietal lobule (IPL, extending to the left temporo-parietal junction, TPJ; Figure 3). In addition, increased brain activity was also detected in dopaminergic-rich sub-cortical regions (e.g., bilateral thalamus, right putamen, and right caudate nucleus; Table 2) known to be involved in somatosensory integration, motivation, goal-directed actions as well as formation habits and procedural memory (Ashby and Crossley, 2010, 2012). On the other hand, the brain activity associated with correct behavioral predictions of non-initially intended serves (compared to initially intended serves) revealed only one specific hemodynamic increase in the left calcarine gyrus, extending to the left cuneus-a brain region associated with visual information processing.
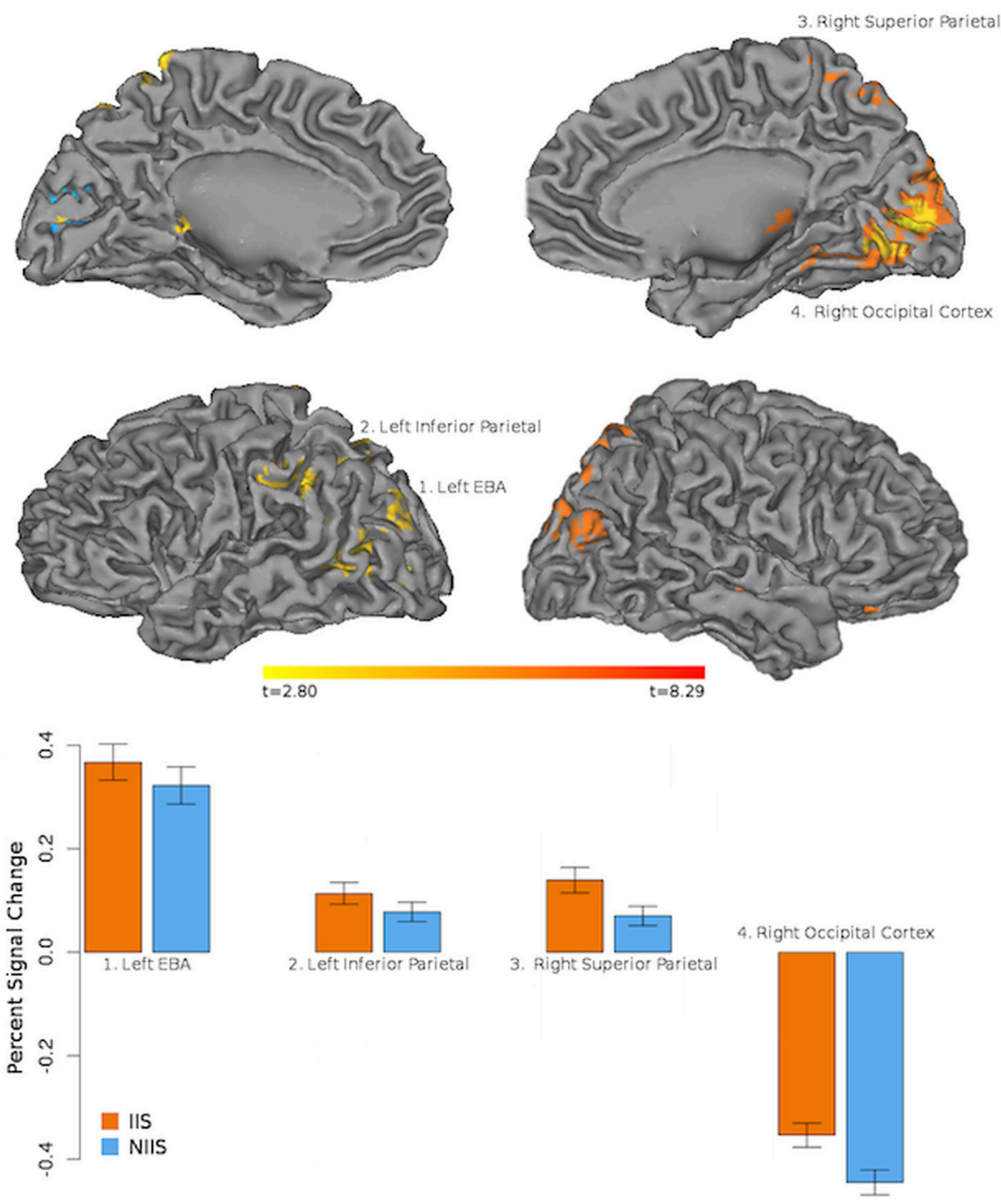

FIGURE 3 | Neuroimaging results representing the comparison between the IIS and NIIS contrasts for correct trials. Results are projected onto the Caret AFNI Colin Brain surface model. Voxelwise threshold at $p<.01$, multiple comparison correction to minimum cluster vol of $702 \mathrm{ul}$ ( 26 voxels). Results from the IIS - NIIS contrast are represented in orange/red. Results from the NIIS - IIS contrast are represented in blue. 
Table 2 | BOLD responses obtained for IIS > NIIS for correct trials.

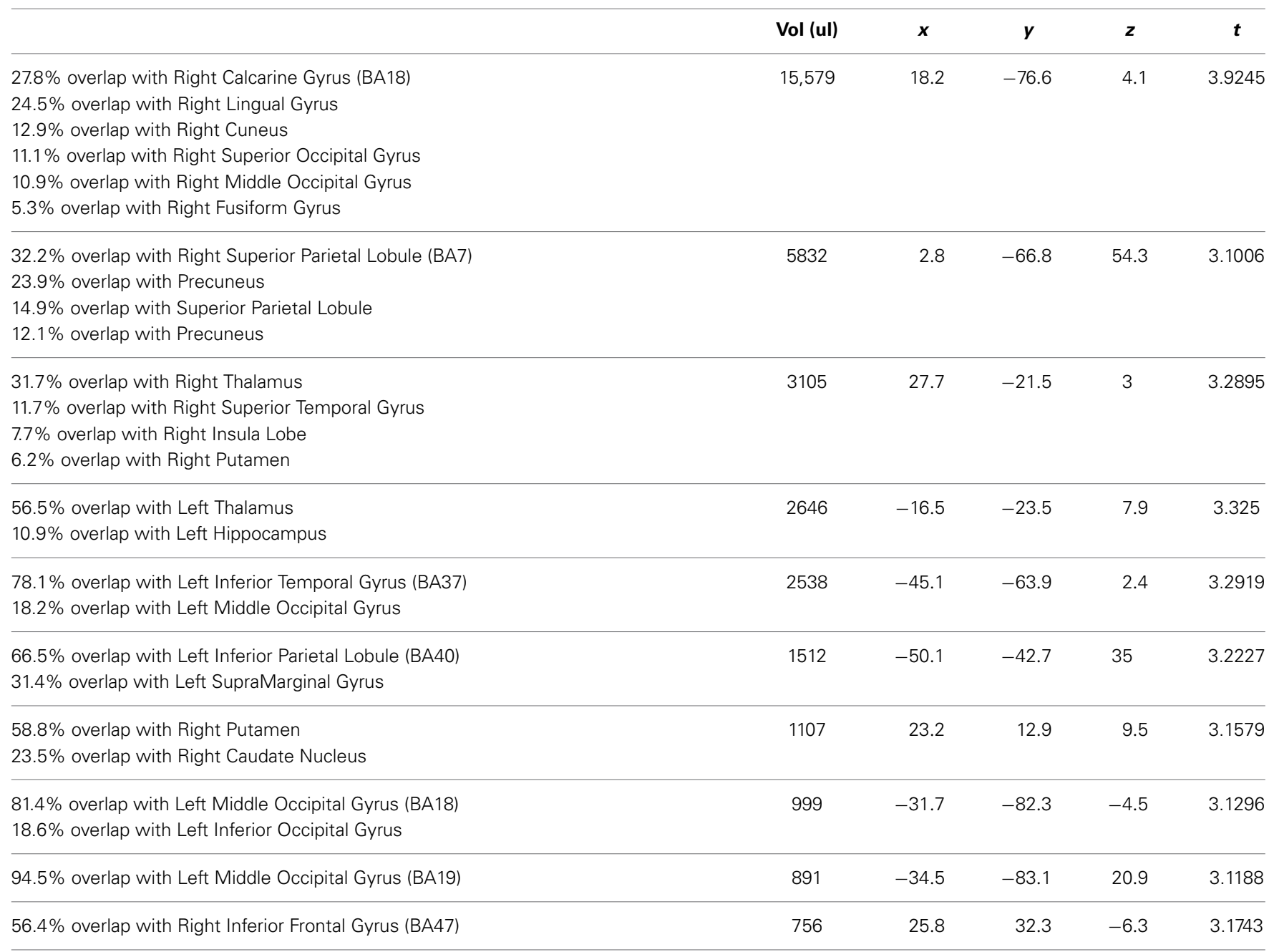

The overlap is the percentage of voxels relative to the entire size of the cluster that overlapped with a given region's spatial map in the AFNI MNI atlas. So, for example, "32.2\% overlap in Right Superior Parietal Lobule" means 32.2\% of the cluster's entire volume was spatially located in the MNI region outlining the Right Superior Parietal Lobule.

To further determine whether the above brain areas were specific to correct trials of the $t \mathrm{IIT}$, we also performed an analysis of the brain activity of the participants' incorrect trials. As for the analyses of correct trials, the neuroimaging analyses of the regional changes in brain activity for incorrect behavioral predictions of IIS (compared to NIIS) revealed hemodynamic activation in brain areas involved in basic analysis of visual information and biological motion (right occipital cortex), attention (right SPL), and simulation and action observation (e.g., left inferior frontal gyrus, inferior parietal lobule; Table 3; Figure 4).

Similar to the correct trials, the brain activity associated with incorrect behavioral predictions of non-initially intended serves (compared to initially intended serves) revealed only one specific hemodynamic increase in the left cuneus extending to the left calcarine gyrus - a brain region associated with visual information processing. Different from the neuroimaging results for the correct trials, incorrect trials were also characterized by brain activity in right TPJ (right superior temporal gyrus) and left precentral gyrus/inferior frontal, and right IPL, but not in the right inferior frontal gyrus nor left IPL (Table 3; Figure 4). Indeed, compared to correct trials, incorrect trials were characterized by the absence of activations between IIS and NIIS in: (i) the caudate or thalamus, (ii) the right inferior frontal gyrus (an area known to be important in intention understanding; Iacoboni et al., 2005), (iii) left IPL, and (iv) EBA area.

\section{fMRI correlational analyses}

For correct trials, a positive correlation was observed between the accuracy index and BOLD IIS-NIIS contrast signal in dopaminergic-rich brain areas involved in procedural memory (such as the caudate nucleus; Figure 5), in the cerebellum and the left middle/superior temporal gyrus (Table 4), two areas known to be involved in analyses of motor movements and social cognition (Van Overwalle et al., 2014). Interestingly, correlations between $d^{\prime}$ accuracy index and BOLD IIS-NIIS contrast revealed a different pattern of regional brain activations (Table S2). This 
Table 3 | BOLD responses obtained for IIS > NIIS for incorrect trials.

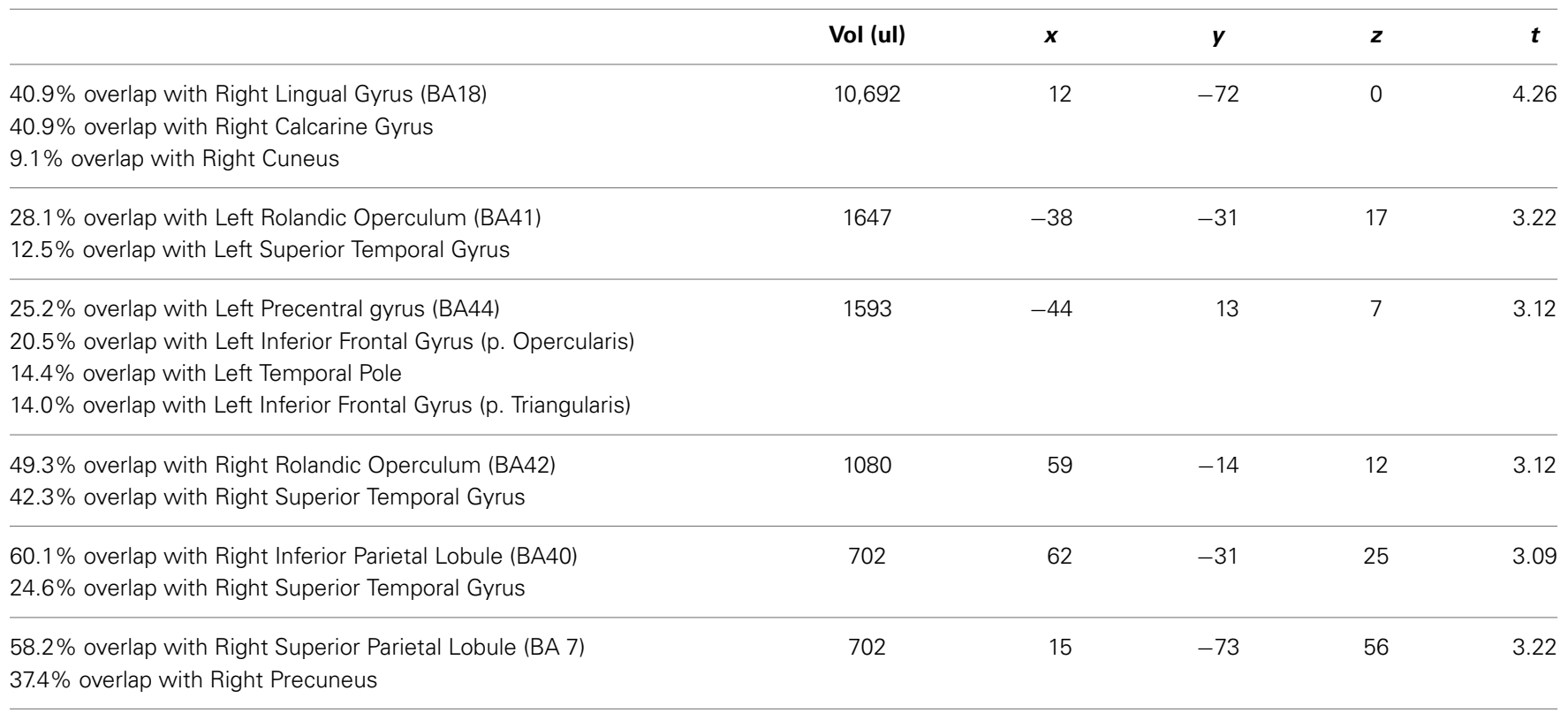

The overlap is the percentage of voxels relative to the entire size of the cluster that overlapped with a given region's spatial map in the AFNI MNI atlas. So, for example, "40.9\% overlap in Right Lingual Gyrus" means $40.9 \%$ of the cluster's entire volume was spatially located in the MNI region outlining the Right Lingual Gyrus.

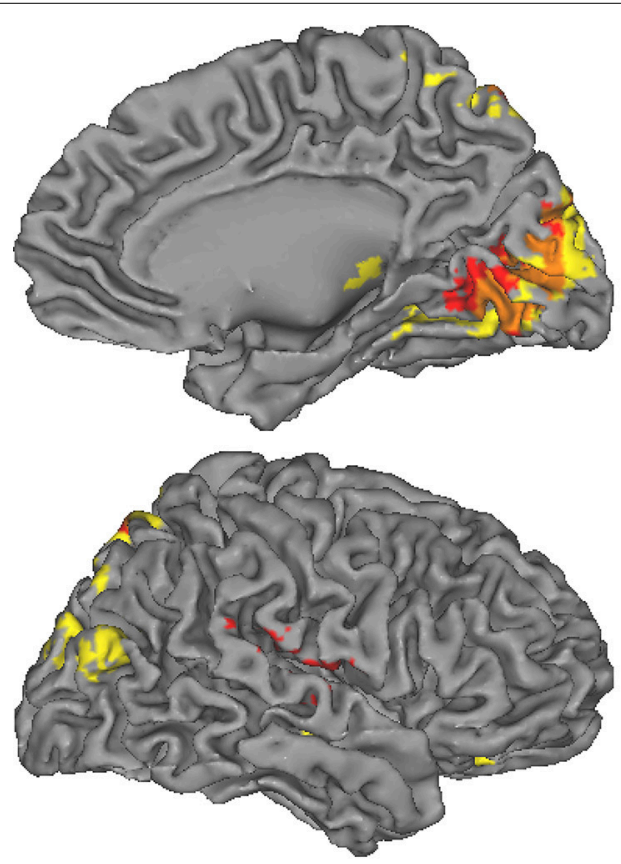

IIS $>$ NIIS, Correct Responses - IIS $>$ NIIS, Incorrect Responses - IIS $>$ NIIS, Correct / Incorrect Overlap

FIGURE 4 | Neuroimaging results representing the comparison between the IIS and NIIS contrast effects. A surface projection on the Caret AFNI Colin Brain surface model depicting the two-tailed result of the main effects for IIS - NIIS for both correct and incorrect trials ( $p<0.01$, corrected). Color codes indicate cluster effects for correctly identified trials (yellow: IIS > NIIS, green: NIIS > IIS) and incorrectly identified trials (red: IIS > NIIS, blue: NIIS >

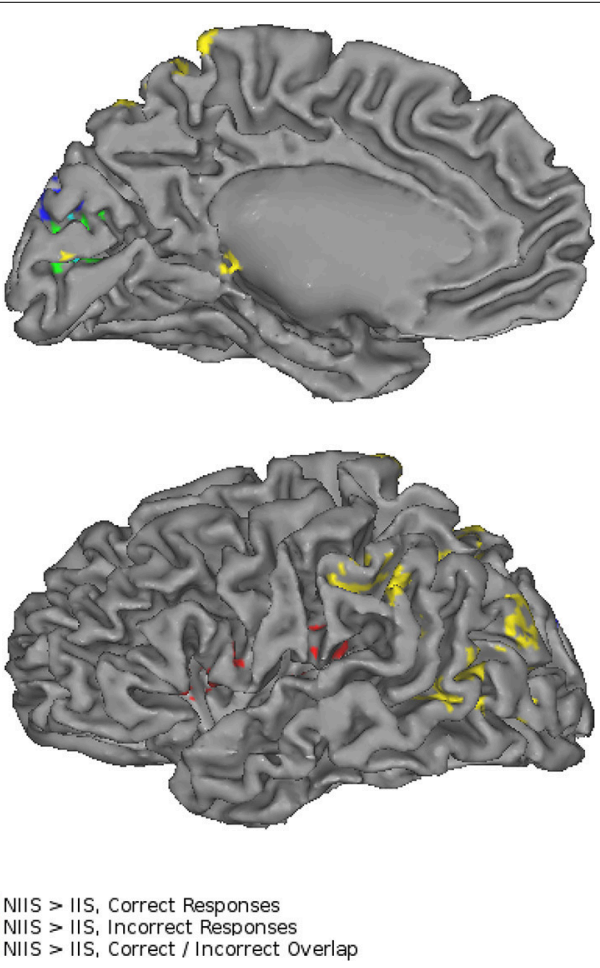

IIS). Overlaps between these results are indicated with a combination of colors: orange regions indicate where correct and incorrect responses overlapped for IIS > NIIS contrasts in the right cuneus, while cyan indicates overlap of correct and incorrect NIIS > IIS effects in the left cuneus. No regions showed overlap between different directions of effects (i.e., IIS > NIIS and NIIS > IIS or vice-versa). 


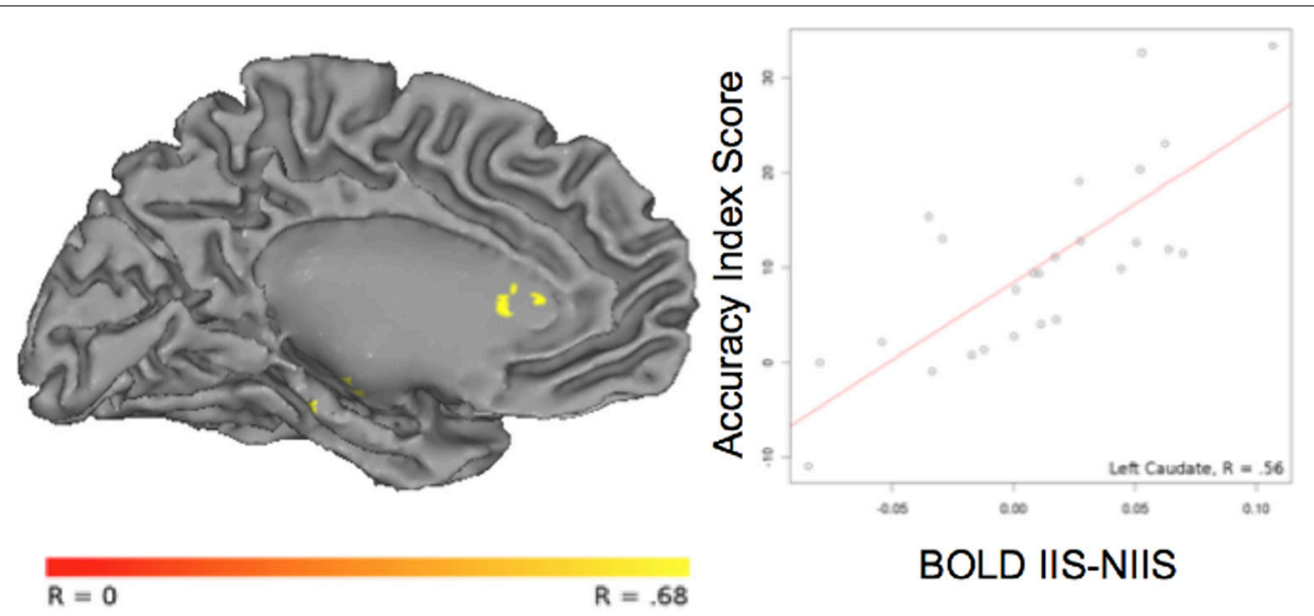

FIGURE 5 | Correlation between BOLD activity and behavioral accuracy index in the left caudate nucleus. The correlation between the contrast of BOLD [IIS - NIIS] $\times$ accuracy index score $(r=0.56, p<.01$, corrected $)$ is projected onto the Caret AFNI Colin Brain surface model.

Table 4 | Correlations between BOLD [IIS - NIIS] for correct trials and behavioral measures.

\begin{tabular}{|c|c|c|c|c|c|}
\hline BOLD [IIS - NIIS] for correct trials $x$ Accuracy index & Vol (ul) & $\boldsymbol{x}$ & $y$ & $z$ & $r$ \\
\hline $\begin{array}{l}\text { 15.1\% overlap with Cerebellum } \\
8.8 \% \text { overlap with Cerebellar Vermis } \\
8.4 \% \text { overlap with Cerebellar Vermis }\end{array}$ & 3105 & 0 & -30 & -21 & 0.57 \\
\hline $\begin{array}{l}57.7 \% \text { overlap with Left Middle Temporal Gyrus (BA21) } \\
\text { 9.5\% overlap with Left Superior Temporal Gyrus }\end{array}$ & 2268 & -47 & -30 & -2 & 0.57 \\
\hline BOLD [IIS - NIIS] for correct trials $\times$ Reaction times & Vol (ul) & $x$ & $y$ & $z$ & $r$ \\
\hline
\end{tabular}

NO CLUSTERS FOUND

The overlap is the percentage of voxels relative to the entire size of the cluster that overlapped with a given region's spatial map in the AFNI MNI atlas. So, for example, "15.1\% overlap in Cerebellum" means $15.1 \%$ of the cluster's entire volume was spatially located in the MNI region outlining the Cerebellum.

is in line with $d^{\prime}$ and simple accuracy measures reflecting different psychological mechanisms. More precisely, the accuracy index reflects correct trials whether by stimulus discriminability or response biases, whereas $d^{\prime}$ accuracy reflects stimulus discriminability per se. Although both indices were significant for the superior temporal gyrus, correlational results for the $d^{\prime}$ accuracy index selectively revealed greater activation in brain areas involved in attention, intention understanding (e.g., inferior frontal gyrus), perceptual discrimination (e.g., hypothalamus), and associative memory and perception of places and visual paths (e.g., parahippocampal region; Rajimehr et al., 2011; Table S2). Finally, no significant correlation was observed between the reaction times and BOLD IIS-NIIS contrast for correct trials (Table 4).

For incorrect trials, no significant correlations were observed between the accuracy index and BOLD IIS-NIIS contrast (Table 5), whereas a negative correlation was found in the left angular gyrus for the $d^{\prime}$ accuracy index (Table S3). This result suggests that the more participants made incorrect discriminations between the two conditions, the less this part of the brain was activated. The present results suggest that a reduced activity of this brain area, which is known to sustain various functions, such as self-other expansion, embodied cognition, and mental representation of past self-experiences, may play an important role in the commission of errors in tennis serve predictions. Finally, significant correlations were found for reaction times (Table 5). These correlations were a positive correlation in the right superior frontal gyrus and a negative correlation in the right parahippocampal gyrus (Table 5).

USTA levels. A positive correlation was observed between USTA levels and BOLD activity for correct trials in the calcarine gyrus, extending to the lingual gyrus, left post-central gyrus, left thalamus, left caudate nucleus, right superior temporal gyrus, bilateral hippocampus, right para-hippocampal region, bilateral precuneus, left IFG, bilateral IPL, right angular gyrus, anterior cingulate and SMA (Table 6). Although no correlation was found 
Table 5 | Correlations between BOLD [IIS - NIIS] for incorrect trials and behavioral measures.

\begin{tabular}{|c|c|c|c|c|c|}
\hline BOLD [IIS - NIIS] for incorrect trials $\times$ Accuracy index & Vol (ul) & $\boldsymbol{x}$ & $y$ & $z$ & $r$ \\
\hline & \multicolumn{5}{|c|}{ NO CLUSTERS FOUND } \\
\hline $52.2 \%$ overlap with Right Superior Frontal Gyrus (BA8) & 1809 & 4 & 34 & 44 & 0.56 \\
\hline $51.0 \%$ overlap with Right Parahippocampal Gyrus (BA 30) & 1026 & 30 & -58 & 6 & -0.57 \\
\hline
\end{tabular}

between behavioral results and USTA levels, these findings are interesting as they suggest that the higher the USTA level of the participants was, the more intense was the activity in these brain regions involved in goal-directed motor actions, embodied cognition, attention, intention understanding, self-other expansion, and associative and procedural memory. No significant clusters were found between the USTA levels of the participants and their brain activity recruited during the difference scores of incorrect trials between IIS and NIIS (Table 7).

Number of hours playing tennis per week. A negative correlation was observed between this measure and the activity in a brain area involved in risk taking and decision making and simulation (insula; Paulus et al., 2003) for the difference scores of correct trials between IIS and NIIS (Table 6). These findings are preliminary but may suggest that the more participants reported playing tennis per week, the less the activity in these two brain areas when making correct tennis serve predictions-an interpretation that is consistent with the behavioral correlations we reported above.

For the difference scores of incorrect trials between IIS and NIIS, a negative correlation $(r=-0.59, p<.05)$ was observed in the right superior temporal gyrus (BA 41; Table 7), suggesting that the more participants reported playing tennis per week, the less the activity in this particular brain areas when making incorrect tennis serve predictions.

Number of hours watching tennis on TV per week. No significant clusters were found between the number of hours participants reported watching tennis on TV and their brain activity recruited during the difference scores of correct trials between IIS and NIIS (Table 6). For incorrect predictions, however, a significant negative correlation was observed between this measure and the activity in their right angular gyrus (Table 7), which suggests that the more participants reported watching tennis on TV, the less their right angular gyrus was activated during their incorrect predictions. These results are consistent our above results suggesting a potential important role of the de-activation (or reduced activity) of the angular gyrus in incorrect tennis serve predictions. Finally, other negative correlations between the reported number of hours watching tennis on TV per week and activity in brain areas involved in motion, intention understanding, attention, and goal-directed actions (See Table 7 for details).
Age of the participants when they first begun playing tennis. For correct trials, a negative correlation was observed between this tennis-related measure and brain activity in the right pallidum, right caudate nucleus, and bilateral insula, whereas a positive correlation was observed between this measure and the activity in the right medial frontal gyrus, and brain areas involved in self-other representation, simulation and embodied cognition (see Table 6 for details). For incorrect trials, negative correlations were observed in the right insula, and bilateral superior temporal gyrus, although positive correlations were observed in the right angular gyrus, the right hippocampus, right supramarginal gyrus, and the right cerebellum (Table 7).

\section{DISCUSSION}

Anticipating intentions of an opponent during a fast interaction is a challenging problem. Our results reinforce and expand prior research by demonstrating that tennis experts are better at predicting where an expert server intends to serve ( $T$ or wide) when that expert server knows where he intends to serve before than after he tosses the ball in the air. Because the same action (a serve) can reflect different intentions (e.g., to serve to the $\mathrm{T}$ or the wide side of the service box) in tennis, the present study highlights the power of cognitive thinking prior action in interpersonal intention understanding. Although the participants were not able to explicitly articulate the reason of their successful tennis serve predictions, they were better at predicting IIS than NIIS.

These findings support predictions by the simulation and embodied cognition theories by demonstrating that the observers are more efficient in predicting one's intentions when that someone is pre-cognizant of their intentions before initiating their action (IIS condition) than when they don't know in advance their action intention (NIIS condition). In other words, when the observers share a common mental representation of action with the server, observers can more accurately read the intentions of the server. As demonstrated in previous research, this facilitation effect in reading another's intentions is positively correlated with active practice (as measured with the number of hours playing tennis per week) rather than passive practice (as measured with the number of hours watching tennis per week). Further studies could be done to specifically test the effects of the different components of a tennis profile and habits of a player on their anticipatory behaviors and performance. For instance, based on simulation and embodied cognition theories, one may be interested in comparing the effect of active tennis practice (e.g., 
Table 6 | Correlations between tennis-related, self-reported measures, and BOLD [IIS - NIIS] contrast for correct trials.

\begin{tabular}{lcccc}
\hline BOLD [IIS - NIIS] x USTA level & Vol (ul) & $\boldsymbol{x}$ & $\boldsymbol{y}$ & $\boldsymbol{z}$ \\
\hline $10.4 \%$ overlap with Left Calcarine Gyrus & 47,466 & -4 & -46 & -1
\end{tabular}

8.0\% overlap with Left Lingual Gyrus

$6.4 \%$ overlap with Right Lingual Gyrus

$5.2 \%$ overlap with Left Calcarine Gyrus

$3.9 \%$ overlap with Left Fusiform Gyrus

3.9\% overlap with Left Middle Occipital Gyrus

$3.8 \%$ overlap with Left Hippocampus

$21.5 \%$ overlap with Left Postcentral Gyrus

9558

$-25$

$-17$

28

0.56

9.4\% overlap with Left SupraMarginal Gyrus,

$5.6 \%$ overlap with Left Thalamus,

$5.4 \%$ overlap with Left Caudate Nucleus

$4.6 \%$ overlap with Left Middle Frontal Gyrus

$3.3 \%$ overlap with Left Inferior Parietal Lobule

$34.9 \%$ overlap with Right Superior Temporal Gyrus

17.9\% overlap with Right Hippocampus

$10.6 \%$ overlap with Right ParaHippocampal Gyrus

$6.8 \%$ overlap with Right Middle Temporal Gyrus

$5.4 \%$ overlap with Right Fusiform Gyrus

$3.3 \%$ overlap with Right Insula Lobe

$17.1 \%$ overlap with Right Parietal Lobe/Precuneus (BA 7)

3240

27

$-65$

$16.3 \%$ overlap with Right Angular Gyrus

$14.7 \%$ overlap with Right Superior Occipital Gyrus

$14.6 \%$ overlap with Right Precuneus

$13.7 \%$ overlap with Right Cuneus

3.8\% overlap with Right Middle Temporal Gyrus

3.4\% overlap with Right Superior Parietal Lobule

$44.2 \%$ overlap with Right Postcentral Gyrus (BA3)

2619

43

$-20$

41

0.55

$34.6 \%$ overlap with Right Precentral Gyrus

3.5\% overlap with Right Inferior Parietal Lobule

33.8\% overlap with Right Middle Cingulate Gyrus (BA 24)

$31.9 \%$ overlap with Right SMA

1917

6

0

46

0.54

$13.7 \%$ overlap with Right SMA

9.0\% overlap with Middle Cingulate Cortex

4.4\% overlap with Right Superior Frontal Gyrus

83.9\% overlap with Left Parietal Lobule/Precuneus (BA 7)

$11.0 \%$ overlap with Left Inferior Parietal Lobule

4.1\% overlap with Left Precuneus

24.2\% overlap with Left Anterior Cingulate Cortex (BA32)

3.6\% overlap with Left Inferior Frontal Gyrus

$80.8 \%$ overlap with Right Precentral Gyrus (BA4)

$15.0 \%$ overlap with Right Rolandic Operculum

\section{BOLD [IIS - NIIS] x Hours playing tennis per week}

$65.2 \%$ overlap with Left Insula Lobe (BA 13)

$33.5 \%$ overlap with Left Inferior Frontal Gyrus

BOLD [IIS - NIIS] x Hours watching tennis per week 1566

$-22$

$-61$

52

0.54 999

$-23$

33

$12 \quad 0.57$

918

58

58

$-9$

22

Vol (ul)

729

29

\begin{tabular}{cccc}
$\boldsymbol{x}$ & $\boldsymbol{y}$ & $\boldsymbol{z}$ & $\boldsymbol{r}$ \\
-35 & 21 & 12 & -0.57 \\
$\boldsymbol{x}$ & $\boldsymbol{y}$ & $\boldsymbol{z}$ & $\boldsymbol{r}$ \\
\hline
\end{tabular}

NO CLUSTERS FOUND 
Table 6 | Continued

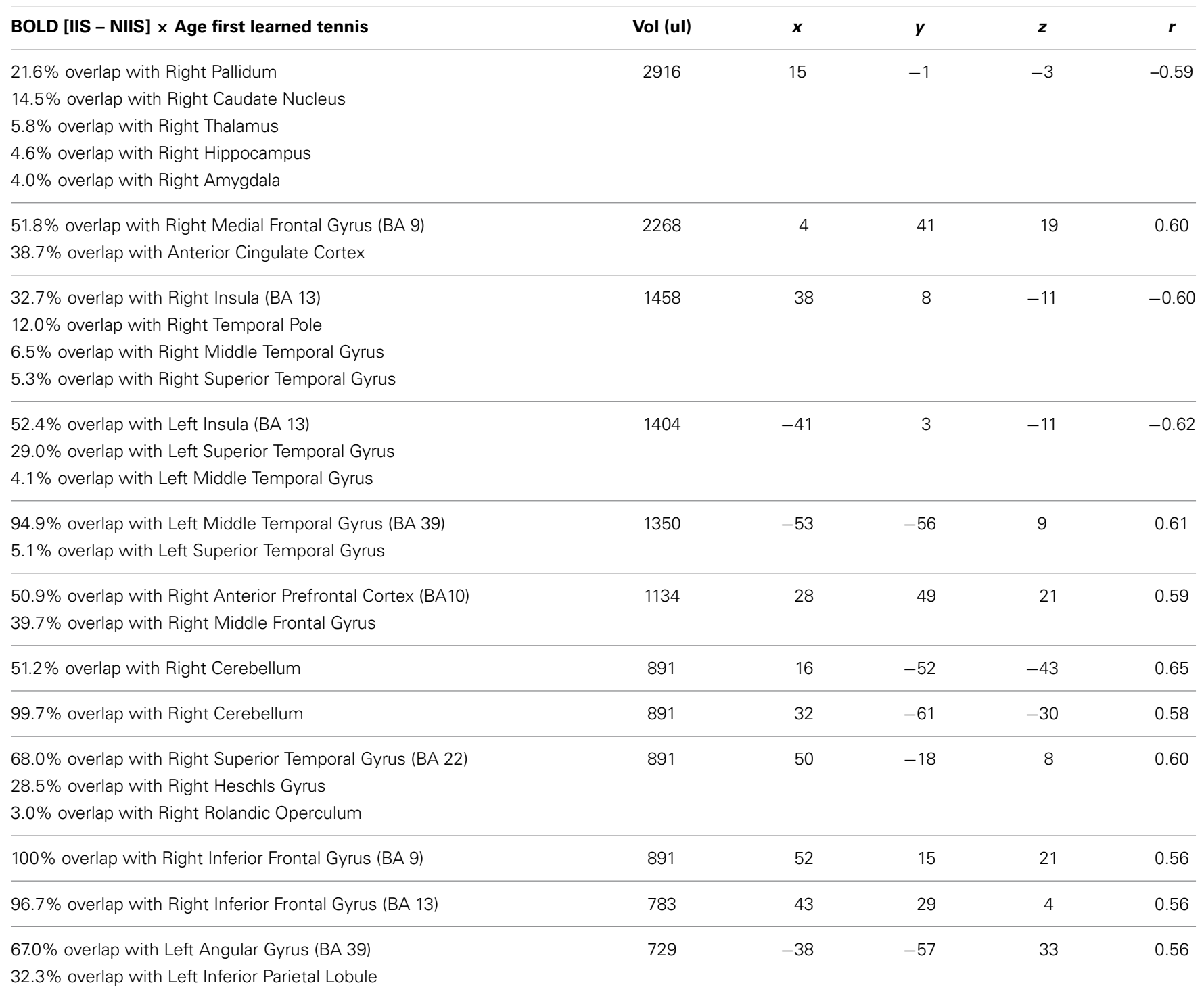

The overlap is the percentage of voxels relative to the entire size of the cluster that overlapped with a given region's spatial map in the AFNI MNI atlas. So, for example, "67.0\% overlap in Left Angular Gyrus" means $67.0 \%$ of the cluster's entire volume was spatially located in the MNI region outlining the Left Angular Gyrus.

playing tennis every week) vs. passive tennis practice (e.g., observing tennis on TV every week) on the accuracy and speed of serve predictions.

Our neuroimaging results extend these behavioral findings by demonstrating that accurate predictions were characterized by activation within both the Action Observation Network (AON including the hMNS) and the social brain network (SN). More precisely, our fMRI analyses of the IIS-NISS contrast for correct trials revealed activation of brain areas known to be involved in a broad variety of functions, including: (a) action prediction tasks (EBA in basketball athletes; Abreu et al., 2012), perception of body parts (Downing et al., 2001), limb movements, motor imagery and performance of motor action (Astafiev et al., 2004); (b) basic analysis of visual information and biological motion (calcarine gyrus, lingual gyrus; Servos et al., 2002); (c) attention and target discrimination (e.g., SPL; Capotosto et al., 2013); (d) retrieval of sensorimotor information and episodic memory (e.g., inferior parietal lobule; Sestieri et al., 2013); (e) action perspective from an egocentric viewpoint and intention understanding; (f) embodied cognition and simulation (e.g., inferior frontal gyrus, inferior parietal lobule; Grafton, 2009; Ortigue et al., 2009a, 2010a; Juan et al., 2013; Mazzarella et al., 2013); and (g) analysis of biological motion, agency, body parts, and perspective taking (STS and inferior parietal lobule; Buchel et al., 1998; Grezes et al., 2001; Ruby and Decety, 2001, 2003, 2004; Grossman and Blake, 2002; Ruby et al., 2002; Astafiev et al., 2004; Wright and Jackson, 2007). By demonstrating such a specific involvement of the inferior frontoparietal network for correct trials (no similar constellation of brain activation was observed for incorrect trials; see Table 2), our present results reinforce Wright and Jackson (2007)'s results 
Table 7 | Correlations between tennis-related, self-reported measures, and BOLD [IIS - NIIS] contrast for incorrect trials.

\begin{tabular}{|c|c|c|c|c|c|}
\hline BOLD [IIS - NIIS] x USTA level & Vol (ul) & $x$ & $y$ & $z$ & $r$ \\
\hline & \multicolumn{5}{|c|}{ NO CLUSTERS FOUND } \\
\hline $41.0 \%$ overlap with Right Superior Temporal Gyrus (BA41) & 1161 & 42 & -38 & 9 & -0.59 \\
\hline $\begin{array}{l}\text { 49.6\% overlap with Right Angular Gyrus (BA 39) } \\
\text { 19.7\% overlap with Right Middle Temporal Gyrus } \\
7.1 \% \text { overlap with Right Middle Occipital Gyrus }\end{array}$ & 5184 & 41 & -60 & 22 & -0.57 \\
\hline $\begin{array}{l}38.0 \% \text { overlap with Left Cerebellum } \\
22.8 \% \text { overlap with Left Cerebellum } \\
12.7 \% \text { overlap with Left Fusiform Gyrus }\end{array}$ & 3672 & -25 & -60 & -33 & -0.55 \\
\hline $\begin{array}{l}\text { 91.7\% overlap with Right Middle Frontal Gyrus (BA 9) } \\
7.2 \% \text { overlap with Right Superior Frontal Gyrus }\end{array}$ & 2349 & 32 & 35 & 37 & -0.59 \\
\hline $\begin{array}{l}\text { 30.5\% overlap with Left Posterior Cingulate Cortex (BA 29) } \\
16.7 \% \text { overlap with Cerebellar Vermis } \\
10.7 \% \text { overlap with Left Calcarine Gyrus }\end{array}$ & 2052 & -3 & -49 & 6 & -0.63 \\
\hline $\begin{array}{l}\text { 82.0\% overlap with Right Superior Parietal Lobule (BA 7) } \\
\text { 9.3\% overlap with Right Postcentral Gyrus }\end{array}$ & 1890 & 33 & -55 & 57 & -0.58 \\
\hline $\begin{array}{l}\text { 32.4\% overlap with Left Parahippocampal Area (BA 34) } \\
11.3 \% \text { overlap with Left Putamen }\end{array}$ & 945 & -24 & 1 & -9 & -0.6 \\
\hline $\begin{array}{l}3.8 \% \text { overlap with Right Postcentral Gyrus (BA 2) } \\
1.5 \% \text { overlap with Right SupraMarginal Gyrus }\end{array}$ & 864 & 31 & -23 & 37 & -0.55 \\
\hline $\begin{array}{l}\text { 88.4\% overlap with Left Superior Temporal Gyrus (BA 13) } \\
11.6 \% \text { overlap with Left SupraMarginal Gyrus }\end{array}$ & 810 & -54 & -40 & 19 & -0.6 \\
\hline BOLD [IIS - NIIS] x Age first learned tennis & Vol (ul) & $x$ & $y$ & $z$ & $r$ \\
\hline $\begin{array}{l}21.4 \% \text { overlap with Right Insula } \\
\text { 13.7\% overlap with Right Amygdala } \\
5.4 \% \text { overlap with Right Putamen }\end{array}$ & 1809 & 22 & 6 & -10 & -0.63 \\
\hline $\begin{array}{l}54.1 \% \text { overlap with Right Angular Gyrus (BA 39) } \\
\text { 31.0\% overlap with Right Middle Occipital Gyrus } \\
7.2 \% \text { overlap with Right Middle Temporal Gyrus }\end{array}$ & 1809 & 42 & -70 & 29 & 0.56 \\
\hline
\end{tabular}


Table 7 | Continued

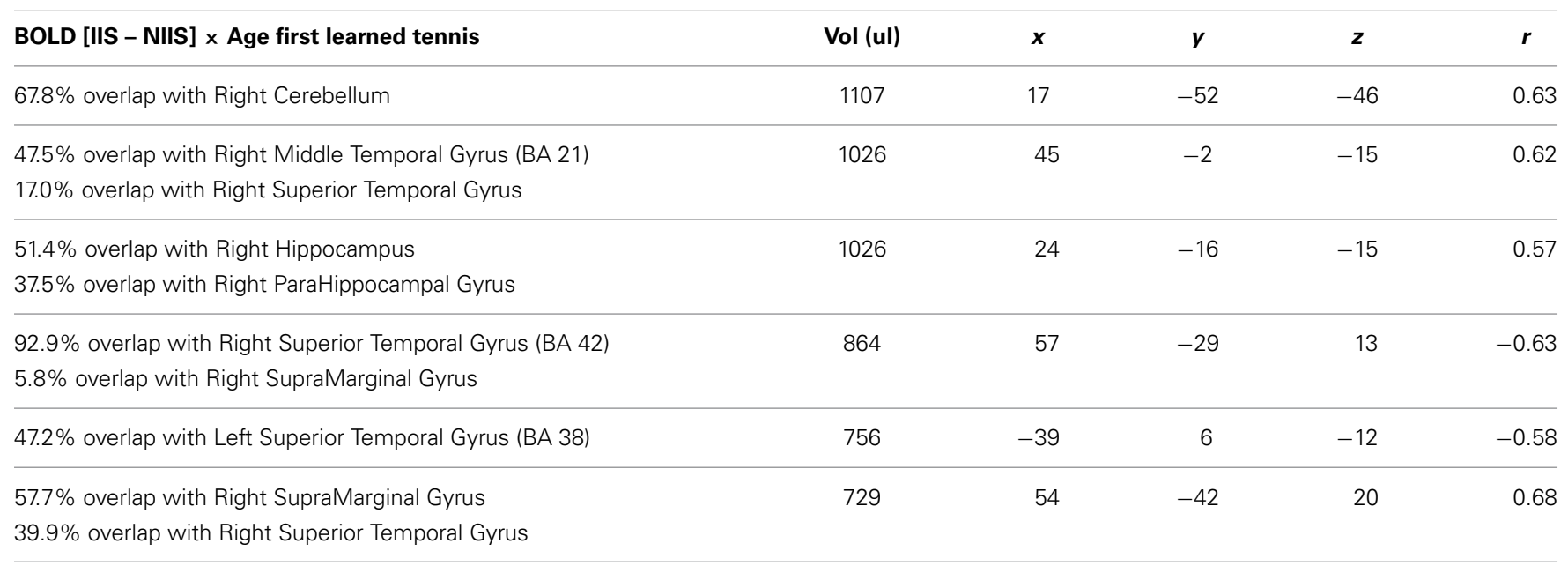

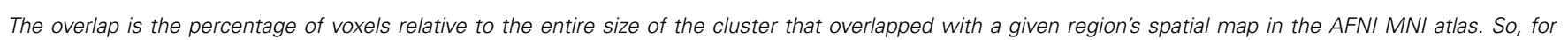

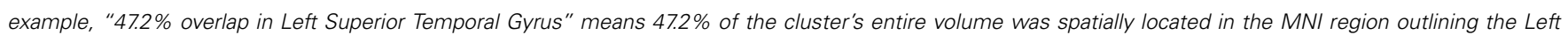
Superior Temporal Gyrus.

by highlighting the role of hMNS in tennis action prediction, and also reinforce simulation and embodied cognition theories (Grafton, 2009; Ortigue et al., 2009a, 2010a; Juan et al., 2013; Mazzarella et al., 2013), which suggest that reactivation of brain areas involved in own's motor performance and integration of past self experiences can facilitate fast and automatic visuo-motor matching process between what the observer sees and what they have executed (over and over) in the past.

Furthermore, the specific pattern of activation for correct IIS (compared to NIIS) suggests that accurate identification of a server's ultimate intentions regarding the direction of their serves operates through top-down processes as it builds on brain areas that have been previously recruited during observation and performance (practice) of that serve, adding regions associated with habit formation, body feature detection and performance. This interpretation also fits with the positive correlations found between some of these brain areas and the USTA levels. The closer the USTA level of the participants was to that of the model in the video, the more intense the activity was in these brain regions. To further investigate these correlations between USTA levels and top-down processes from these brain areas, future studies could be done with models having very high (expert) vs. very low (non-expert) USTA levels. Including these models with different USTA levels would allow to dissociate between motor and visual expertise.

In addition, IIS (compared to NIIS) was characterized by increased activity in dopaminergic-rich regions (bilateral thalamus, right putamen, right insula, and right caudate nucleus) involved in somatosensory integration, motivation, goal-directed actions as well as formation habits and procedural memory (Ashby and Crossley, 2010, 2012). The specific recruitment of a striatal based procedural memory in the understanding of intended serves is of particular interest as it reinforces previous studies highlighting the importance of procedural memory in embodied cognition and intention understanding (e.g., Altmann and Trafton, 2002; Grafton, 2009). Because the dorsal parts of the striatum, such as the caudate and putamen, are innervated by dopamine coming from both the ventral tegmental area and substantia nigra and going out to the insula also track rewarding stimuli of conditioned incentive value, the present results suggest that the recruitment of the dorsal striatum may be critical for the convergence between sensorimotor integration experience during both the practice of a tennis serve and the rewarding experience of predicting correctly an opponent's serve during prior matches. Further studies need to be done to test this hypothesis.

Finally, incorrect trials were associated with a different configuration of brain activation, which may provide clues as to when intention prediction goes wrong. Although overlapping areas of activations were observed within the brain areas involved in basic visual processing and spatial attention, no activation was observed during incorrect trials in brain areas involved in action prediction, embodied cognition, and procedural memory. Our study instead reveals that inaccurate predictions are related to activation in cortical areas known to be involved in low-level (bottom-up) computational processes associated with the sense of agency and self-other distinction as well as high-level processes such as theory of mind (Decety and Lamm, 2007). Notably, a specific activation was observed for incorrect trials in the right TPJ-a heteromodal association cortical area, emcompassing the supramarginal gyrus, posterior superior temporal gyrus, and the dorsal part of the occipital gyrus, which is involved both in lowlevel computational processes associated with theory of mind, perspective taking, a sense of agency, and higher social cognitive tasks (Decety and Lamm, 2007) and self-other distinction (Decety and Sommerville, 2003; Decety and Lamm, 2007). Further studies using high temporal resolution imaging methods (such as high density electrical neuroimaging) may help delineate the specific spatio-temporal dynamics of this brain area during inaccurate predictions of others' intentions. However, together the results of our study suggest experienced tennis players may make more 
accurate predictions of the service intentions of a skilled opponent when they focus on the somatic representation they observe rather than mentalizing about the strategy and explicit service intentions of their opponent.

\section{ACKNOWLEDGMENTS}

This study was supported by a professorship grant from the Swiss National Science Foundation (FNS\# PP00_1_128599/1 to SC). The authors thank Aaron B. Ball, Stephen Balogh and Ryan Paradise for their technical help, as well as Professor Brian Martens for his early comments on the stimuli.

\section{SUPPLEMENTARY MATERIAL}

The Supplementary Material for this article can be found online at: http://www.frontiersin.org/journal/10.3389/fnhum. 2014.00781/abstract

Movie 1 | IIS stimulus sample. Example of a video clip in which the tennis player was told the intended location of his serve before he began his serve preparation.

Movie 2 | NIIS stimulus sample. Example of a video clip in which the tennis player did not know in advance the location of his serve. Rather the experimenter told him where to serve when the ball was in the air.

\section{REFERENCES}

Abernethy, B., and Zawi, K. (2007). Pickup of essential kinematics underpins expert perception of movement patterns. J. Mot. Behav. 39, 353-367. doi: 10.3200/JMBR.39.5.353-368

Abreu, A. M., Macaluso, E., Azevedo, R. T., Cesari, P., Urgesi, C., and Aglioti, S. M. (2012). Action anticipation beyond the action observation network: a functional magnetic resonance imaging study in expert basketball players. Euro. J. Neurosci. 35, 1646-1654. doi: 10.1111/j.1460-9568.2012.08104.x

Aglioti, S. M., Cesari, P., Romani, M., and Urgesi, C. (2008). Action anticipation and motor resonance in elite basketball players. Nat. Neurosci. 11, 1109-1116. doi: $10.1038 / \mathrm{nn} .2182$

Agnew, C. R., and Etcheverry, P. E. (2006). "Cognitive interdependence and relationship commitment: self-in-relationship," in Intrapersonal Processes and Interpersonal Relationships: How They Relate, eds K. D. Vohs and E. J. Finkel (New York, NY: Guilford Press), 274-293.

Allison, T., Puce, A., and McCarthy, G. (2000). Social perception from visual cues: role of the STS region. Trends Cogn. Sci. 4, 267-278. doi: 10.1016/S13646613(00)01501-1

Altmann, E. M., and Trafton, J. G. (2002). Memory for goals: an activation-based model. Cogn. Sci. 26, 39-83. doi: 10.1207/s15516709cog2601_2

Ashby, G. F., and Crossley, M. J. (2012). Automaticity and multiple memory systems. Wiley Interdiscip. Rev. 3, 363-376. doi: 10.1002/wcs.1172

Ashby, G. F., and Crossley, M. J. (2010). Interactions between declarative and procedural-learning categorization systems. Neurobiol. Learn. Mem. 94, 1-12. doi: 10.1016/j.nlm.2010.03.001

Astafiev, S. V., Stanley, C. M., Shulman, G. L., and Corbetta, M. (2004). Extrastriate body area in human occipital cortex responds to the performance of motor actions. Nat. Neurosci. 7, 542-548. doi: 10.1038/nn1241

Bellini, L. M., Baime, M., and Shea, J. A. (2002). Variation of mood and empathy during internship. JAMA J. Am. Med. Assoc. 287, 3143-3146. doi: 10.1001/jama.287.23.3143

Blakemore, S. J., and Decety, J. (2001). From the perception of action to the understanding of intention. Nat. Rev. Neurosci. 2, 561-567. doi: 10.1038/35086023

Buccino, G., Cossu, G., De Fanti, A., Manotti, C., Izzi, G. C., and Mancia, D. (2004). Cerebral venous sinus thrombosis in childhood: clinical aspects and neurological and cognitive long-term outcome in three cases. Neurol. Sci. 25, 296-300. doi: 10.1007/s10072-004-0357-6

Buchel, C., Price, C., and Friston, K. (1998). A multimodal language region in the ventral visual pathway. Nature 394, 274-277. doi: 10.1038/28389
Calvo-Merino, B., Glaser, D. E., Grèzes, J., Passingham, R. E., Haggard, P., and Grezes, J. (2005). Action observation and acquired motor skills: an FMRI study with expert dancers. Cereb. Cortex 15, 1243-1249. doi: 10.1093/cercor/bhi007

Capotosto, P., Tosoni, A., Spadone, S., Sestieri, C., Perrucci, M. G., Romani, G. L., et al. (2013). Anatomical segregation of visual selection mechanisms in human parietal cortex. J. Neurosci. 33, 6225-6229. doi: 10.1523/JNEUROSCI.498312.2013

Cross, E., Kraemer, D., Hamilton, A. F., Kelley, W., and Grafton, S. (2009). Sensitivity of the action observation network to physical and observational learning. Cereb. Cortex 19, 315-326. doi: 10.1093/cercor/bhn083

Cross, E. S., Hamilton, A. F., and Grafton, S. G. (2006). Building a motor simulation de novo: observation of dance by dancers. Neuroimage 31, 1257-1267. doi: 10.1016/j.neuroimage.2006.01.033

Cutting, J. E., and Kozlowski, L. T. (1977). Recognizing friends by their walk: gait perception without familiarity cues. Bull. Psychon. Soc. 9, 353-356. doi: 10.3758/BF03337021

Davis, M. H. (1980). A multidimensional approach to individual differences in empathy. JSAS Cat. Sel. Doc. Psychol. 10, 85.

Decety, J., and Cacioppo, S. (2012). The speed of morality: a highdensity electrical neuroimaging study. J. Neurophysiol. 108, 3068-3072. doi: 10.1152/jn.00473.2012

Decety, J., and Lamm, C. (2007). The role of the right temporoparietal junction in social interaction: how low-level computational processes contribute to metacognition. Neuroscientist 13, 580-593. doi: 10.1177/1073858407304654

Decety, J., and Sommerville, J. A. (2003). Shared representations between self and others: a social cognitive neuroscience view. Trends Cogn. Sci. 7, 527-533. doi: 10.1016/j.tics.2003.10.004

Desmurget, M., and Grafton, S. (2000). Forward modeling allows feedback control for fast reaching movements. Trends Cogn. Sci. 4, 423-431. doi: 10.1016/S13646613(00)01537-0

Desmurget, M., Reilly, K. T., Richard, N., Szathmari, A., Mottolese, C., and Sirigu, A. (2009). Movement intention after parietal cortex stimulation in humans. Science 324, 811-813. doi: 10.1126/science.1169896

Downing, P. E., Jiang, Y., Shuman, M., and Kanwisher, N. (2001). A cortical area selective for visual processing of the human body. Science 293, 2470-2473. doi: $10.1126 /$ science. 1063414

Farrow, D., and Abernethy, B. (2003). Do expertise and the degree of perceptionaction coupling affect natural anticipatory performance? Perception 32, 1127-1139. doi: 10.1068/p3323

Frith, C. D., and Frith, U. (1999). Interacting minds - a biological basis. Science 286, 1692-1695. doi: 10.1126/science.286.5445.1692

Glencross, D., and Cibich, B. (1977). A decision analysis of games skills. Aust. J. Sports Med. 9, 72-75.

Goulet, C., Bard, C., and Fleury, M. (1992). Attentional requirements for preparing to return a serve in tennis. Can. J. Sport Sci. 17, 98-103.

Goulet, C., Fleury, M., Bard, C., Yerlès, M., Michaud, D., and Lemire, L. (1988). Analysis of advance visual indices in receiving a tennis serve. Can. J. Sport Sci. 13, 79-87.

Grafton, S. T. (2009). Embodied cognition and the simulation of action to understand others. Ann. N.Y. Acad. Sci. 1156, 97-117. doi: 10.1111/j.17496632.2009.04425.x

Grafton, S. T., Arbib, M. A., Fadiga, L., and Rizzolatti, G. (1996). Localization of grasp representations in humans by positron emission tomography. 2 . Observation compared with imagination. Exp. Brain Res. 112, 103-111. doi: 10.1007/BF00227183

Grafton, S. T., Aziz-Zadeh, L., and Ivry, R. (2009). "Hierarchy in action representation," in The New Cognitive Neurosciences, Vol. IV, ed M. S. Gazzaniga (Cambridge, MA: MIT press), 641-655.

Grezes, J., Fonlupt, P., Bertenthal, B., Delon-Martin, C., Segebarth, C., and Decety, J. (2001). Does perception of biological motion rely on specific brain regions? Neuroimage 13, 775-785. doi: 10.1006/nimg.2000.0740

Grossman, E. D., and Blake, R. (2002). Brain areas active during visual perception of biological motion. Neuron 35, 1167-1175. doi: 10.1016/S0896-6273(02) 00897-8

Helsen, W., and Starkes, J. (1999). A multidimensional approach to skilled perception and performance in sport. Appl. Cogn. Psychol. 13, 1-27.

Holmes, C. J., Hoge, R., Collins, L., Woods, R., Toga, A. W., and Evans, A. C. (1998). Enhancement of MR images using registration for signal averaging. J. Comput. Assist. Tomogr. 22, 324-333. doi: 10.1097/00004728-199803000-00032 
Hommel, B., Müsseler, J., Aschersleben, G., and Prinz, W. (2001). The Theory of Event Coding (TEC): a framework for perception and action planning. Behav. Brain Sci. 24, 849-937. doi: 10.1017/S0140525X01000103

Iacoboni, M., Molnar-Szakacs, I., Gallese, V., Buccino, G., Mazziotta, J. C., and Rizzolatti, G. (2005). Grasping the intentions of others with one's own mirror neuron system. PLoS Biol. 3:e79. doi: 10.1371/journal.pbio.0030079

Jackson, R. C., and Mogan, P. (2007). Advance visual information, awareness, and anticipation skill. J. Mot. Behav. 39, 341-351. doi: 10.3200/JMBR.39.5. $341-352$

Jeannerod, M. (2001). Neural simulation of action: a unifying mechanism for motor cognition. Neuroimage 14, 103-109. doi: 10.1006/nimg.2001.0832

Juan, E., Frum, C., Bianchi-Demicheli, F., Wang, Y.-W., Lewis, J. W., and Cacioppo, S. (2013). Beyond human intentions and emotions. Front. Hum. Neurosci. 7:99. doi: 10.3389/fnhum.2013.00099

Kilner, J. M., Friston, K. J., and Frith, C. D. (2007). Predictive coding: an account of the mirror neuron system. Cogn. Process. 8, 159-166. doi: 10.1007/s10339-0070170-2

Kilner, J. M., and Frith, C. D. (2007). A possible role for primary motor cortex during action observation. Proc. Natl. Acad. Sci. U.S.A. 104, 8683-8684. doi: 10.1073/pnas.0702937104

Kording, K. P., and Wolpert, D. M. (2004). Bayesian integration in sensorimotor learning. Nature 427, 244-247. doi: 10.1038/nature02169

Lamm, C., Batson, C. D., and Decety, J. (2007). The neural substrate of human empathy: effects of perspective-taking and cognitive appraisal. J. Cogn. Neurosci. 19, 42-58. doi: 10.1162/jocn.2007.19.1.42

Marshall, J. C., Caplan, D., and Holmes, J. M. (1975). The measure of laterality. Neuropsychologia 13, 315-321. doi: 10.1016/0028-3932(75)90008-1

Mazzarella, E., Ramsey, R., Conson, M., and Hamilton, A. (2013). Brain systems for visual perspective taking and action perception. Soc. Neurosci. 8, 248-267. doi: 10.1080/17470919.2012.761160

Nichols, T. E. (2012). Multiple testing corrections, nonparametric methods, and random field theory. Neuroimage 62, 811-815. doi: 10.1016/j.neuroimage.2012. 04.014

Niedenthal, P. M. (2007). Embodying emotion. Science 316, 1002-1005. doi: 10.1126/science. 1136930

Niedenthal, P. M., Barsalou, L. W., Winkielman, P., Krauth-Gruber, S., and Ric, F. (2005). Embodiment in attitudes, social perception, and emotion. Pers. Soc. Psychol. Rev. 9, 184-211. doi: 10.1207/s15327957pspr0903_1

Oldfield, R. C. (1971). The assessment and analysis of handedness: the Edinburgh inventory. Neuropsychologia 9, 97-113. doi: 10.1016/0028-3932(71)90067-4

Ortigue, S., King, D., Gazzaniga, M., Miller, M., and Grafton, S. (2009a). Right hemisphere dominance for understanding the intentions of others: evidence from a split-brain patient. BMJ Case Rep. 2009, 6-11. doi: 10.1136/bcr.07.2008.0593

Ortigue, S., Patel, N., Bianchi-Demicheli, F., and Grafton, S. T. (2010b). Implicit priming of embodied cognition on human motor intention understanding in dyads in love. J. Soc. Pers. Relat. 27, 1001-1015. doi: 10.1177/02654075 10378861

Ortigue, S., Sinigaglia, C., Rizzolatti, G., and Grafton, S. T. (2010a). Understanding Actions of others: the electrodynamics of the left and right hemispheres. A high-density EEG neuroimaging study. PLoS ONE 5:e12160. doi: 10.1371/journal.pone. 0012160

Ortigue, S., Thompson, J. C., Parasuraman, R., and Grafton, S. T. (2009b). Spatiotemporal dynamics of human intention understanding in temporo-parietal cortex: a combined EEG/fMRI repetition suppression paradigm. PLoS ONE 4:e6962. doi: 10.1371/journal.pone.0006962

Overney, L. S., Blanke, O., and Herzog, M. H. (2008). Enhanced temporal but not attentional processing in expert tennis players. PLoS ONE 3:e2380. doi: 10.1371/journal.pone. 0002380

Paulus, M. P., Rogalsky, C., Simmons, A., Feinstein, J. S., and Stein, M. B. (2003). Increased activation in the right insula during risk-taking decision making is related to harm avoidance and neuroticism. Neuroimage 19, 1439-1448. doi: 10.1016/S1053-8119(03)00251-9

Rajimehr, R., Devaney, K. J., Bilenko, N. Y., Young, J. C., and Tootell, R. B. H. (2011). The "parahippocampal place area" responds preferentially to high spatial frequencies in humans and monkeys. PLoS Biol. 9:e1000608. doi: 10.1371/journal.pbio. 1000608

Rizzolatti, G., and Craighero, L. (2004). The mirror-neuron system. Annu. Rev. Neurosci. 27, 169-192. doi: 10.1146/annurev.neuro.27.070203.144230
Rizzolatti, G., Fadiga, L., Gallese, V., and Fogassi, L. (1996). Premotor cortex and the recognition of motor actions. Brain Res. Cogn. Brain Res. 3, 131-141. doi: 10.1016/0926-6410(95)00038-0

Rizzolatti, G., and Fogassi, L. (2014). The mirror mechanism: recent findings and perspectives. Philos. Trans. R. Soc. Lond. B Biol. Sci. 369, 1471-2970. doi: 10.1098/rstb.2013.0420

Rizzolatti, G., and Sinigaglia, C. (2007). Mirror neurons and motor intentionality. Funct. Neurol. 22, 205-210.

Rizzolatti, G., and Sinigaglia, C. (2008). Further reflections on how we interpret the actions of others. Nature 455, 589. doi: 10.1038/455589b

Ruby, P., and Decety, J. (2001). Effect of subjective perspective taking during simulation of action: a PET investigation of agency. Nat. Neurosci. 4, 546-550. doi: $10.1038 / 87510$

Ruby, P., and Decety, J. (2003). What you believe versus what you think they believe: a neuroimaging study of conceptual perspective-taking. Eur. J. Neurosci. 17, 2475-2480. doi: 10.1046/j.1460-9568.2003.02673.x

Ruby, P., and Decety, J. (2004). How would you feel versus how do you think she would feel? A neuroimaging study of perspective-taking with social emotions. J. Cogn. Neurosci. 16, 988-999. doi: 10.1162/0898929041502661

Ruby, P., Sirigu, A., and Decety, J. (2002). Distinct areas in parietal cortex involved in long-term and short-term action planning: a PET investigation. Cortex 38, 321-339. doi: 10.1016/S0010-9452(08)70663-4

Ruscher, J. B., Santuzzi, A. M., and Hammer, E. Y. (2003). Shared impression formation in the cognitively interdependent dyad. Br. J. Soc. Psychol. 42, 411-425. doi: $10.1348 / 014466603322438233$

Russell, D. (1996). UCLA loneliness scale (Version 3): reliability, validity, and factor structure. J. Pers. Assess. 66, 20-40. doi: 10.1207/s15327752jpa6601_2

Servos, P., Osu, R., Santi, A., and Kawato, M. (2002). The neural substrates of biological motion perception: an fMRI study. Cereb. Cortex 12, 772-782. doi: 10.1093/cercor/12.7.772

Sestieri, C., Capotosto, P., Tosoni, A., Luca Romani, G., and Corbetta, M. (2013). Interference with episodic memory retrieval following transcranial stimulation of the inferior but not the superior parietal lobule. Neuropsychologia 51, 900-906. doi: 10.1016/j.neuropsychologia.2013.01.023

Shim, J., Les Carlton, G., and Kwon, Y. H. (2006). Perception of kinematic characteristics of tennis strokes for anticipating stroke type and direction. Res. Q. Exerc. Sport 77, 326-339. doi: 10.1080/02701367.2006.10599367

Singer, R. N., Cauraugh, J. H., Chen, D., Steinberg, G. M., and Frehlich, S. G. (1996). Visual search, anticipation, and reactive comparisons between highlyskilled and beginning tennis players. J. Appl. Sport Psychol. 8, 9-26. doi: 10.1080/10413209608406305

Spielberger, C. D. (1987). State-trait anxiety inventory. Anxiety 19, 2009

Tenenbaum, G., Sar-El, T., and Bar-Eli, M. (2000). Anticipation of ball location in low and high-skill performers: a developmental perspective. Psychol. Sport Exerc. 1, 117-128. doi: 10.1016/S1469-0292(00)00008-X

Tomeo, E., Cesari, P., Aglioti, S. M., and Ugesi, C. (2013). Fooling the kickers but not the goalkeepers: behavioral and neurophysiological correlates of fake action detection in soccer. Cereb. Cortex 23, 2765-2778. doi: 10.1093/cercor/bhs279

Van Overwalle, F., Baetens, K., Mariën, P., and Vandekerckhove, M. (2014). Social cognition and the cerebellum: a meta-analysis of over 350 fMRI studies. Neuroimage 86, 554-572. doi: 10.1016/j.neuroimage.2013.09.033

Ward, B. D. (2002). Deconvolution Analysis of fMRI Time Series Data. Milwaukee, WI: Biophysics Research Institute, Medical College of Wisconsin.

Ward, P., Williams, A. M., and Bennett, S. (2002). Perception of relative motion in tennis. Res. Q. Exerc. Sport 73, 107-112. doi: 10.1080/02701367.2002.10608997

Wegner, D. M., Giuliano, T., and Hertel, P. T. (1985). "Cognitive interdependence in close relationships," in Compatible and Incompatible Relationships, ed W. J. Ickes (New York, NY: Springer), 253-276.

Wheatley, T., Milleville, S. C., and Martin, A. (2007). Understanding animate agents: distinct roles for the social network and mirror system. Psychol. Sci. 18, 469-474. doi: 10.1111/j.1467-9280.2007.01923.x

Williams, A. M., Huys, R., Cañal-Bruland, R., and Hagemann, N. (2009). The dynamical information underpinning anticipation skill. Hum. Mov. Sci. 28, 362-370. doi: 10.1016/j.humov.2008.10.006

Williams, A. M., Ward, P., Knowles, J. M., and Smeeton, N. J. (2002). Anticipation skill in a real-world task: measurement, training, and transfer in tennis. J. Exp. Psychol. Appl. 8, 259-270. doi: 10.1037/1076-898X.8.4.259

Williams, A. M., Ward, P., Smeeton, N. J., and Allen, D. (2004). Developing anticipation skills in tennis using on-court instruction: perception 
versus perception and action. J. Appl. Sport Psychol. 16, 350-360. doi: $10.1080 / 10413200490518002$

Wolpert, D. M., Doya, K., and Kawato, M. (2003). A unifying computational framework for motor control and social interaction. Philos. Trans. R. Soc. Lond. B Biol. Sci. 358, 593-602. doi: 10.1098/rstb.2002.1238

Wright, M. J., and Jackson, R. C. (2007). Brain regions concerned with perceptual skills in tennis: an fMRI study. Int. J. Psychophysiol. 63, 214-220. doi: 10.1016/j.ijpsycho.2006.03.018

Zigmond, A. S., and Snaith, R. P. (1983). The hospital anxiety and depression scale. Acta. Psychiatr. Scand. 67, 361-370. doi: 10.1111/j.1600-0447.1983.tb09716.x

Conflict of Interest Statement: The authors declare that the research was conducted in the absence of any commercial or financial relationships that could be construed as a potential conflict of interest.
Received: 26 April 2014; accepted: 15 September 2014; published online: 06 October 2014.

Citation: Cacioppo S, Fontang F, Patel N, Decety J, Monteleone G and Cacioppo JT (2014) Intention understanding over $T:$ a neuroimaging study on shared representations and tennis return predictions. Front. Hum. Neurosci. 8:781. doi: 10.3389/fnhum. 2014.00781

This article was submitted to the journal Frontiers in Human Neuroscience. Copyright (C) 2014 Cacioppo, Fontang, Patel, Decety, Monteleone and Cacioppo. This is an open-access article distributed under the terms of the Creative Commons Attribution License (CC BY). The use, distribution or reproduction in other forums is permitted, provided the original author(s) or licensor are credited and that the original publication in this journal is cited, in accordance with accepted academic practice. No use, distribution or reproduction is permitted which does not comply with these terms. 